Max-Planck-Institut für demografische Forschung

Max Planck Institute for Demographic Research

Doberaner Strasse 114 - D-18057 Rostock · GERMANY

Tel +49 (0) 3812081 - 0; Fax +49 (0) 3812081 - 202;

http://www.demogr.mpg.de

MPIDR WORKING PAPER WP 2002-034

AUGUST 2002

\title{
Demographic composition and projections of car use in Austria
}

\author{
Alexia Prskawetz (fuernkranz@ demogr.mpg.de) \\ Jiang Leiwen \\ Brian C. O'Neill
}

(c) Copyright is held by the authors.

Working papers of the Max Planck Institute for Demographic Research receive only limited review.

Views or opinions expressed in working papers are attributable to the authors and do not necessarily reflect those of the Institute. 


\title{
Demographic composition and projections of car use in Austria ${ }^{1}$
}

\author{
Alexia Prskawetz ${ }^{2 \cdot 3}$ \\ Max Planck Institute for Demographic Research, Rostock, Germany \\ Jiang Leiwen \\ Institute of Population Research, Peking University \\ and \\ Watson Institute for International Studies, Brown University, USA \\ Brian C. O’Neill \\ International Institute for Applied Systems Analysis, Laxenburg, Austria \\ and \\ Watson Institute for International Studies, Brown University, USA
}

\begin{abstract}
Understanding the factors driving demand for transportation in industrialized countries is important in addressing a range of environmental issues. Though noneconomic factors have received less attention, recent research has found that demographic factors are important. While some studies have applied a detailed demographic composition to analyze past developments of transportation demand, projections for the future are mainly restricted to aggregate demographic variables such as numbers of people and/or households. In this paper, we go beyond previous work by combining cross-sectional analysis of car use in Austria with detailed household projections. We show that projections of car use are sensitive to the particular type of demographic disaggregation employed. For example, the highest projected car use - an increase of about 20 per cent between 1996 and 2046 - is obtained if we apply the value of car use per household to the projected numbers of households. However, if we apply a composition that differentiates households by size, age and sex of the household head, car use is projected to increase by less than 3 per cent during the same time period.
\end{abstract}

Keywords: household projections, car use demand, demographic composition, Austria

\footnotetext{
${ }^{1}$ This paper was partly written while Jiang Leiwen and Brian C. O’Neill were visiting the Max Planck Institute for Demographic Research in autumn 2000 and in winter 2002. The authors are grateful for the help provided by Zeng Yi and Wang Zhenglian in appyling the household projection program ProFamy and for comments and suggestions by participants and in particular by the discussant Anna Babette Wils at the session on 'Population-Environment in Urban Settings' at the PAA 2002 meeting in Atlanta. For language editing, we would like to thank Michael Garrett and Susann Baker.

${ }^{2}$ Corresponding author: e-mail:fuernkranz@demogr.mpg.de, phone: +49(0) 3812081 141, fax: +49(0) 381 2081441.

${ }^{3}$ The views expressed in this paper are the author's views and do not necessarily reflect those of the Max Planck Institute for Demographic Research.
} 


\section{Introduction}

Understanding the factors driving demand for transportation in industrialized countries is important in addressing a range of environmental issues including local air pollution and climate change (NRC, 1997). Understanding is also an aid to planners who must anticipate infrastructure needs and address congestion concerns. Research on travel demand and transportation fuel use has shown that demand generally rises with income (e.g., Dahl and Sterner, 1991). Non-economic factors have received less attention but have been found to be important. Links between indicators of lifestyle and energy use have been identified (Schipper et al., 1989). Analyses of household survey data in the U.S. have shown differences in travel demand across households that differ in the age and gender of the householder, household size and composition, and family type (Pucher et al., 1998; O'Neill and Chen, 2002). Carlsson-Kanyama and Linden (1999) find similar relationships in Sweden, showing that women, the elderly, and those with low incomes generally travel less than men, the middle-aged, and those with higher incomes. In addition to the consideration of separate demographic variables, the life-cycle concept has been demonstrated to provide a useful framework for capturing variation in travel demand and associated greenhouse gas emissions across households that differ by some combination of family size, family type, age of the householder, and marital status (Greening and Jeng, 1994; Greening et al., 1997). Other studies have shown that household characteristics are not only important in explaining variation in travel demand, but also in anticipating household response to price changes or other policies (Kayser, 2000).

Little work has focused on the role demographic characteristics of households might play in explaining past changes in aggregate demand, or to predict future changes. O'Neill and Chen (2002) use a standardized procedure to conclude that changes in household size, age, and composition in the U.S. over the past several decades have likely had a substantial influence on aggregate demand for direct energy use by households. Buettner and Grubler (1995) point out that sex-specific cohort effects on car ownership in Germany are likely to be quite significant and will influence future travel demand as populations age. Spain (1997) finds a similar pattern in the U.S., where far more baby boom women hold driver's licenses than the current generation of elderly women, portending an increase in travel demand in elderly age groups in the future.

However, these studies either simply suggest particular demographic variables that may be important in projections, or make transportation projections in the absence of detailed household projections. In this paper, we go beyond previous work by combining crosssectional analysis of car use in Austria with detailed household projections. This approach raises additional methodological questions, because it may be that some characteristics that are important in explaining cross-sectional variation in travel behavior are not important in projecting future demand. This could result if the population composition is not going to shift across demographic categories that may be important in explaining variation in transportation behavior (e.g., even if small households travel much less than large ones, projections that ignore this difference will not be subject to 
aggregation error if the proportion of large to small households remains constant in the future).

Our study is divided into three steps. We start with a descriptive analysis of the demographic composition of car use in Austria in 1997. We then perform a detailed household projection for Austria up to the year 2046. We apply these projections to study the change in demographic compositions across time. Finally, we combine car use patterns in 1997 (as decomposed by selected demographic characteristics) with future changes in these demographic compositions.

By applying this three-step procedure, we aim to explore the following questions: (a) what is the best level of demographic composition for understanding the effect of demographic characteristics on private car use in a cross-sectional analysis?, (b) which level of demographic composition will change the most in the future?, and (c) in light of results for (a) and (b), what level of demographic composition is best for projecting future car use?

\section{Data}

The present study is based on the Austrian micro-census (a quarterly and representative household survey of 1\% of all Austrian dwellings) from June 1996 and June 1997. Each survey provides a core-questionnaire on household demographic characteristics such as total household size, number of children, age, gender, marital status, education and working status of the household head plus housing conditions of the household. The sample size is in the order of approximately 30,000 dwellings, but each quarter an eighth of all addresses is replaced. In the particular case of the micro-census of June 1996 and that of June 1997, the survey consisted of 23,174 and 22,648 un-weighted valid cases respectively (a summary of the June 1996 survey is given in Hanika, 1999; for a more detailed description of the June 1997 survey, see Statistic Austria, 1998). The June 1996 survey includes an additional questionnaire on birth biographies. For this reason it was chosen as the base population for conducting a detailed household projection using the ProFamy model (Zeng et al., 1997). In addition, part of the input necessary to run ProFamy was derived from the Austrian Family and Fertility Survey conducted in 199596 (Doblhammer et al., 1997). For the demographic composition analysis of private car use, we use the June 1997 micro-census including information on energy use in households and private car use. Based on these data it is possible to reconstruct, in part, the travel behavior of private households with their first two cars. In particular, the following characteristics can be defined: (1) car ownership and (2) how many kilometers households drove with their first and, if applicable, their second car in the course of the year before the interview. The fact that information is only available for the first two cars is relatively un- problematic as only $6 \%$ of car owners reported owning more than two cars. Total distance driven may be more problematic since it was self- assessed. 


\section{Demographic composition of car use}

We derive the demographic composition of car use patterns from the Austrian microcensus of June 1997. First, we categorize households according to five compositional variables, or combinations of variables: (1) age of household head, (2) age and sex of household head, (3) size of household, (4) number of adults and children in the household, and (5) age of household head and size of household. For each of these five compositions, we next calculate the mean distance driven by households within each category of the compositional variable. Calculations are based only on those households that recorded a positive travel distance during the year preceding June 1997. For instance, in case of composition (1) we calculate the mean distance driven for households whose head is aged 18-24, 25-29, etc. years old, and who report a non-zero distance traveled in the past year. Since the number of households that recorded a positive distance is a subset (of about 90\%) of those households that own a car, we calculate car ownership across the various levels of each composition in a second step. The results of these calculations are summarized in Figure 1a -1e.

To verify the sensitivity of travel demand patterns to alternative compositions, Table 1 summarizes the results of a simple ANOVA analysis applied to the variable that measures the distance driven with the first two cars for each compositional variable. The F-statistics verify that for all compositional variables, the average distances across the categories differ significantly. A comparison across the proportions of total variance accounted for by each model shows that age and size considered independently are almost equally effective in explaining total variance, while age and size together provide the best combination of variables among the models tested.

[Table 1 about here]

[Figure 1a-1e about here]

\section{Household age $e^{4}$}

Figure 1a shows a distinct age pattern of car ownership and car use. Car ownership increases with the age of the household head and reaches a peak of almost $90 \%$ for the 40-44 year age group. Thereafter, ownership declines and falls below the 50\% mark, beginning with the 70-74 year age group. The pattern of car use is very similar to the car ownership pattern in that car use first increases up to the late middle ages and declines thereafter. These age patterns are driven by several factors. Generally, household size first increases with the age of the household head and starts to decline again at older ages. One-person households account for more than $50 \%$ of households aged $<25$ and $>75$, but for less than 20\% of households aged 35-49. Labor-force participation, and consequently the necessity to commute and means of travel, also vary with the age of the household head. Labor-force participation increases from about $70 \%$ for households aged $<25$ to

\footnotetext{
${ }^{4}$ Hereafter, we use "household age" to mean the age of the household head. Note that cohorts of households defined using this definition of age do not necessarily constitute an identical group of households over time, since reorganizations of membership can add or subtract households from a cohort.
} 
$93 \%$ for households aged $40-44$, then declines to $<10 \%$ for households aged $>65$. Cohort effects may also be involved. Today's middle-and young-aged generation has grown up in times when car ownership has been the norm rather than the exception. As these cohorts age, we may expect to see a disproportionate increase in car ownership and car use patterns among the older generation.

Gender differences in car ownership and car use patterns persist across all ages (Figure 1b). While car ownership is about $20 \%$ lower for female- as compared to male-headed households up to age 50, this difference increases to $45 \%$ for older households (e.g. while only $15 \%$ of female-headed households at age 75-79 own a car, $60 \%$ of male-headed households in the same age group do so ). The divergence in ownership with increasing age may partly be caused by a cohort effect. However, we also observe a clear difference in labor-force participation and household size across age between male- and femaleheaded households. While among male-headed households aged 55-59 years about $61 \%$ of all household heads are in the labor-force, only $26 \%$ of all female household heads in the same age category are employed. Corresponding figures for households aged 40-44 are $94 \%$ and $86 \%$, which is a much smaller gap. Moreover, the percentage of single person households is higher among female-headed households, particularly for the older age groups. $82 \%$ of female-headed households in the age category 70-74 are single person households; the corresponding figure for male-headed households is $13 \%$. At age 25-29 this difference is much smaller, with $47 \%$ of female and $34 \%$ of male households being single households. Both trends, the lower female labor-force participation rate and the higher prevalence of single person households, may partly explain the gender gap in car ownership. Since both differences increase with age, this may also explain the increasing gender gap across age.

While gender difference in car ownership increases with age, car use patterns of femaleand male-headed households become more similar with the age of the household head. The gap in car use at younger ages is most likely driven to a large extent by the fact that female-headed households not only tend to be smaller but are also more likely to be single adult households. For households aged 25-44 that own a car, $49 \%$ of femaleheaded households and only $33 \%$ of male-headed households have a single adult. In contrast, for households aged $>65$, the corresponding figures for female-and maleheaded households are nearly identical (92\% and 95\%, respectively). One might suspect that the fact that the gender gap in car use patterns declines with age is also influenced by narrowing gender gaps in labor-force participation as well as size and/or number of adults among households that own a car. However this hypothesis is not supported by the data.

\section{Household size}

Household size (Figure 1c) positively affects car ownership and car use. Part of the household size effect reflects an age effect. Smaller households are more likely to be headed by younger and older people (rather than the middle-aged) and these are the age groups for which both car ownership and use are lowest (Figure 1a).Car ownership increases most between households of size one and two. For car use, the greatest increase is between households of size two and three. The former result may be explained by an 
age effect. Among single-person households, 19\% are young (25-34 ) and 34\% are old (70-80+) households. The corresponding figures for two-person households are shifted away from older households $-14 \%$ and $22 \%$ respectively. Together with Figure 1a, these compositional changes contribute to the increase in car ownership between one- and twoperson households. The sharp increase in mean distance driven between households of size two and three may be attributed to a compositional change in age. Three- person households are more predominantly middle-aged than are one-and two-person households. For example, $74 \%$ of all three-person households that own a car are headed by persons aged 30-59 (the age category with the highest mean distance driven, Figure 1a), whilst only $58 \%$ and $52 \%$ of one-and two-person households respectively fall into this age category. Moreover, the age definition among two-person households that own a car is generally older. While only $24 \%$ and $26 \%$ of one and three-person households respectively that own a car are in the age group 55-74, the corresponding number for households of size two is $46 \%$.

\section{Household composition}

Household size may be too crude a measure since it aggregates households of the same size, independent of the age of household members. A three-person household may either consist of three adults, two adults and one child, or one adult and two children; each of these combinations might be expected to have different transportation demands. (We use age 18, the age at which a driving license can be obtained in Austria, as the age that distinguishes between adults and children.) Figure 1d represents a composition of car ownership and car use that distinguishes between adults and children. From these figures we may draw the following conclusions. Firstly, adult only households have the highest rates of car use and ownership across all household sizes. Secondly, within a given household size, the presence of one or more children reduces car ownership only for single adult households ( i.e. for households of size two, three and four, we observe a marked decrease in car ownership pattern only if there are one, two or three children present, respectively). In short, single parent households have the second lowest car ownership after single adult households. Since the latter group of households is composed of old-and young-aged households (compare our discussion to Figure 1a and 1c) it is not surprising that single adult households have the lowest car ownership. Thirdly, single parent households also have the lowest car use within each household size. However, while the presence of two or more children does not essentially effect the car ownership pattern for households of size $>4$, it markedly reduces car use.

Our results indicate a strong correlation between age of the household head and household size. Figure 1e therefore presents car use and car ownership patterns across age and household size. From these results we may conclude that the age pattern of transportation demand aggregated over all household sizes mainly reflects the age patterns observed for households of size one and two. Larger sized households generally show a more stable age pattern. This may be explained by the fact that firstly, larger sized households are less likely to be headed by persons of very young or alternatively very old age and secondly, that these households are more likely to be composed of two generation households. In the case of multi-generation households, the age pattern of car 
ownership and car use reflects the mix of the life-cycle transportation demand of several generations. In case of single adult households (more prevalent among smaller household sizes), the age pattern of car use and car ownership is tied to the life-cycle demand pattern of only one generation. Seen from an alternative perspective, Figure 1e also shows that the difference in transportation demand between household sizes varies across the age of the household head. For middle- and particularly older-age groups, the difference in transportation demand between household sizes is most pronounced. Given that we are likely to observe a tendency towards smaller sized households and an ageing population in the future (see section 4), a composition by age as well as household size seems to be appropriate for long term projections of transportation demand.

\section{Household projections}

To understand the influence of key demographic factors on car use in the long term, it is important to apply population and household projections that can provide detailed information on changes in demographic determinants in the future. However, conducting a consistent, simultaneous, dynamic population and household projection has remained difficult for a long time. As stated by Lutz et al. (1994, p. 225), “...there is no feasible way to convert information based on individuals ... directly into information on households. Even if these two different aspects could be matched for the starting year there is no way to guarantee consistent changes in both when patterns are projected into the future". Previous studies on population-environment interactions, particularly those on the development of population and energy use, limit their analysis to separately treating population at the individual and household level. Those attempting to combine household and individual level information apply a static approach, mostly utilizing the well-known "household headship" rate method. However, the link between the headshiprate and underlying demographic parameters is unclear, given the difficulty in incorporating assumptions about future changes in demographic events. Moreover, this approach lumps all other household members into the very heterogeneous category "nonhead". Therefore, it can not provide detailed information on changes in demographic factors that may be important for future energy use projection. A dynamic population and household projection is obviously desirable. The advancement in theories and methods of family demography have improved our capacity to achieve this. Dynamic micro- and macro- household models (e.g. Hammel et al., 1976; van Imhoff and Keilman, 1991; Zeng et al., 1997, 1999) have been developed. Benefiting from methodological advances in multi-state demography, Zeng (1991) constructed a family status life-table by extending Bongaarts (1987) nuclear status life-table model. Building on this family status life-table, the dynamic projection model "ProFamy" has been developed to simultaneously and consistently project future household and population changes which can match our research purposes.

By applying the ProFamy model, we conducted a dynamic household and population projection for Austria for the period 1996-2046. From the 1996 micro-census data we derived the baseline population for running ProFamy. Based on data from the 1995-96 Austrian Fertility and Family Survey (FFS) and the 1996 micro-census, we constructed standard schedules that determined future transitional patterns by age, sex, and marital 
status. Standard schedules not derived from the two sources were obtained from alternative data sources of Statistic Austria. From the 1996 micro-census, FFS and Statistic Austria, we also derived summary measures of the base year to provide information on the number of transitions in the starting year. For the summary measures of future years, we applied the assumptions of the medium variant as suggested in the latest projections of Statistic Austria (Hanika, 2000) for the total fertility by parity, life expectancy, mean age at childbearing and external migration (cf. Table 2). Other parameters, such as marriage, remarriage, cohabiting, divorce, leaving parental home and sex ratio at birth were maintained over the whole projection period. For a detailed introduction to the methodological issue of the household projection see Appendix A.

\section{[Table 2 about here]}

[Figure $2 \mathrm{a}-2 \mathrm{~g}$ about here]

Our projection results indicate a moderate increase in population size and number of households between 1996 and 2035 (Figure 2a), followed by a decrease in both after 2035. Moreover, changes in the number of households will be more pronounced than changes in the population size. From Figure $2 b$, we observe a process of population aging for Austria over the next five decades. The proportion of children will continuously decline and the number of adults will grow faster than the total population in 1996-2035 and decrease slower than the total population later on. However, among adults, the percentage of the elderly will increase. In particular, the elderly aged 75-84 and > 85 are groups whose population share will increase the most.

Population aging also implies that households will age ${ }^{5}$ (i.e. the age of the household head will increase). Figure $2 \mathrm{c}$ clearly shows that the peak of households by age of household head will move from age 30 in 1996 to age 40 in 2005, age 50 in 2015, age 60 in 2025, age 70 in 2035 and around age 80 in 2046. This is mainly due to the aging of baby boomers born in the 1960s.

If we look separately at male-and female- headed households by age of the household head (Figure $2 \mathrm{~d}$ and Figure 2e), we generally observe the same trend towards higher ages of the household head. However, we also notice that the peak age of household heads becomes less visible in future years among male-headed households, due to higher male mortality. By 2046, the number of male-headed households is almost evenly distributed among the late 20 s to early 80 s age groups. Regarding female- headed households, we observe a fluctuating pattern of the peak age of household heads across time. In general, there are two peaks across age for all projection periods; one peak around age 20 and the other around age 70. This pattern reflects the fact that women tend to leave the parental home and marry earlier than men, which creates the first peak at around age 20 . Women

\footnotetext{
${ }^{5}$ In some developing countries, where the extended family is common, population aging does not necessarily lead to "aging" of household heads. Since most parents transfer household title to their son when they get old, the age pattern of household headship rates stays unchanged. In Austria, transition of household heads between generations is not common, therefore, population aging means "aging" of household heads.
} 
also have a longer life expectancy which forms the second peak in the advanced age group. However, there is a third peak in the middle period and this peak shifts towards older ages. This is mainly due to the effect of aging baby boomers. Moreover, for femaleheaded households the peak in early age is almost constant across the projection period while the peak in old age shifts towards older ages. Furthermore, except in the very young age group (15-19 years) and the advanced older age group (70+), the number of male headed-households is always greater than the corresponding number of femaleheaded households.

Given that the number of households is projected to increase faster than the total population in 1996-2035 and to decrease slower in 2035-2046, the average household size is expected to decrease (Figure 2f). The latter will decline from 2.4 in 1996 to 1.95 in 2035 and 1.94 in 2046. Numbers of smaller households (one-person and two-person households) will continuously increase while numbers of larger households (four- and more person households) will decrease. The number of three-person households will increase in the early years of 1996-2010 before decreasing subsequently. This change mainly reflects our assumption that the total fertility rate will increase from 1.42 to 1.5 in the period of 1996-2020, and stay constant at a level of 1.5 after 2020. Even though the fertility rate will increase up to 2020, changes in age structure will drive the number of three-person household down, starting around 2010.

Figure $2 \mathrm{~g}$ presents a projection of households by household size and distinguishes between the number of adults and children for each household size category. The projections show that one- and two-adult households will experience significant and continuous growth over the next five decades, with all of the growth attributable to households without children. Three-adult households will increase initially in 1996-2015 but decrease afterwards. Focusing on changes in households by size and by age of household head, one can see that an increasing number of one and two-adult households will be mainly elderly. Furthermore, the number of households with children will decline with the exception of single parents with one or two children for the period 1996-2005.

\section{Household projections under alternative future demographic scenarios}

Taking into account the uncertainty of future demographic parameters, we also present household projections for alternative developments of mortality, fertility and union dissolution patterns.

In the case of fertility and mortality, we apply the low and high variant as given by Statistic Austria (see Table 3 and Appendix A, summary measure) in addition to the medium level of fertility and mortality applied in Figure 2. For the alternative union dissolution scenarios we cannot refer to any prevailing scenarios. We therefore construct a low and high union dissolution scenario, assuming that Austria follows the Italian (low union dissolution scenario) or the Swedish pattern (high union dissolution scenario) of union dissolution by the year 2046. Between 1996 and 2046 we apply a linear interpolation. More specifically, we refer to the Family and Fertility Survey conducted in several European countries in the 1990s and co-ordinated by the Population Activities 
Unit of the UN Economic Commission for Europe (UN ECE PAU). According to these data, out of 19 European countries (cf. Prskawetz et al. 2002) Swedish women of birth cohort 1952-59 have the highest union dissolution rate by age 35 - about 1.5 times that of their Austrian counterparts. At the other end of the scale, Italian women of the same birth cohort have the lowest union dissolution rate by age 35 - about 0.26 times of that of their Austrian counterparts.

\section{[Table 3 about here]}

[Figure 3a-3c about here]

In Figure 3 we have assembled selected results of household projections based on alternative fertility, mortality and dissolution scenarios. A comparison across projections by population size, number of adults and number of households (Figure 3a) show that predicted population size will be most sensitive to the assumed fertility development. This can be explained by the fact that a change in fertility today has a multiplier effect since children born today will have children themselves in the future. The projected number of adults will initially be sensitive to changes in mortality patterns and only around 2025, when the changes in fertility have worked their way through the ages we can observe the impact of fertility changes on the number of adults as well. Changes in the rate of union dissolution only have an impact on the projected number of households. ${ }^{6}$ In Figure $3 b$ we plot the projected share of households for three age groups of the household head. The share of household heads in each of three broad age groups is not overly influenced by alternative demographic scenarios. We observe a pronounced decrease in the percentage of middle-aged household heads, and an increase in the percentage of old-aged household heads, for each demographic future scenario (i.e. the ageing process in households will not be overly affected even under alternative fertility and mortality assumptions in the future). However, projected changes in household size are more sensitive to alternative scenarios. Figure $3 \mathrm{c}$ illustrates a general increase in oneand two-person households while households of size three or more are declining over time. By definition, the share of one- person households is most sensitive to alternative dissolution scenarios. This result is a combination of higher dissolution rates among couples without children and the fact that after a dissolution, at least for one partner, the new household form will be most likely a one-person household. Households of size two and more are most sensitive to fertility and dissolution scenarios.

From Figure 3 we may conclude that alternative fertility scenarios will primarily effect total population size and the share of households of size two and more. Alternative mortality scenarios will have a strong impact on the projected number of adults. Compared to the fertility scenarios, the impact of mortality changes on the distribution of households by age of household head and size of household will be less pronounced. Changes in the dissolution patterns will mainly influence the projected number of

\footnotetext{
${ }^{6}$ This result mainly follows from our assumptions of future levels of TFR and life expectancy at birth which are the same as for the medium scenarios. We are aware that changes in union dissolution rate may induce important effects on TFR and life expectancy. However since we lack appropriate data we had to pose this assumption.
} 
households and will have a pronounced impact on the distribution of households by size. Overall, alternative demographic scenarios will not revert the trends towards older and smaller sized households. However, a composition of households by size is more sensitive to demographic scenarios as compared to a composition of households by age of the household head.

\section{Projections of transportation demand}

Our cross-sectional analysis shows that household car ownership and use varies substantially with the age and sex of the householder as well as size (particularly for the one to three- person households), and with some aspects of household composition. Oneadult households, especially single parent households, differ from households with two or more adults. Moreover, we found that size- effect is partly caused by changes in age composition across households of various sizes and vice versa. More specifically, while the difference in car ownership and car use across age is most pronounced among households of size one and two, household size is most significant for middle and old aged households.

The household projections demonstrate that the age distribution of householders will become significantly older, household size is likely to shift decisively toward one- and two- person households at the expense of large households. Households without children will account for essentially all of the growth in total numbers of households.

To arrive at a projection of car use by various demographic decompositions, we combine the results of the household projections with the corresponding cross sectional decomposition of car ownership and car use patterns. For each category of a demographic decomposition, we multiply the projected number of households with the car ownership rate and the mean distance driven. We neglect any behavioral changes in transportation demand patterns across various demographic compositions. In other words, this exercise highlights the role of changing demographic structures ${ }^{7}$ but neglects any changes in transportation demand across various demographic groups.

Change in car use under different demographic compositions; medium variant of the household projections

[Figure 4 about here]

In our first step, we apply the medium variant of the household projections and plot the change in car use patterns relative to 1996 for each projection step and each demographic composition (Figure 4). To interpret these results, it is helpful to begin with the projection based on constant per capita car use multiplied by projected population size. This projection ignores any compositional changes in the population and may therefore be regarded as the benchmark for comparison of alternative projections that take into account a compositional change of some kind (e.g., household size or age of household

\footnotetext{
${ }^{7}$ Actually in this study we take into account only a few but not all possible changes in behavior of household formation and dissolution.
} 
head). The degree to which these alternative projections differ from the benchmark can be taken as an indicator of the importance of accounting for the compositional variable used in the alternative projection. The effect of adding additional compositional variables (such as adding gender to age) can be measured by examining whether projections incorporating both variables differ substantially from projections with just the primary variable.

We examine two general groups of alternative projections: (a) those that take age composition (and additional variables) into account, and (b) those that take household size (and additional variables) into account. Accounting for the age structure of household heads, we obtain a projected car use pattern that is substantially different in level and pattern than the benchmark projection (Figure 4), namely that car use increases through 2020 to a level about 12\% higher than the benchmark and then decreases to end up about $4 \%$ higher in 2046. This pattern can be explained by the aging of the baby boom generation (cf. Figure 2c) which implies a movement along the "hump-shaped" car use pattern by age as depicted in Figure 1a - an effect that is missed by the constant percapita benchmark projection. Note that a simpler means of capturing age effects - (a projection based on number of adults multiplied by per adult car use) is not able to fully capture this age effect. While it projects greater car use than the benchmark scenario, due to the faster growth of numbers of adults as compared to total population, it treats all adults as a homogenous group and misses the fact that most of the growth in adults before 2020 will be in age categories with relatively high car use, while growth thereafter will increasingly shift to older age categories with relatively low car use.

Considering the gender of the household head in addition to age yields a slightly higher projected car use compared to the projection based on age alone. This increase is due to the fact that male-headed households have a higher car use than female-headed households. However the effect is small: car use is never more than $3 \%$ higher when gender is taken into account in addition to age.

Accounting for household size alone yields a projection that follows the general trend of the benchmark case but peaks about $4 \%$ higher in 2025. This result is driven by the shift toward smaller household sizes: while smaller households have lower car use than larger households, the increase in the number of smaller households is greater than the decrease in the number of larger households, more than compensating for this effect and leading to a net increase in aggregate car use. ${ }^{8}$ A simpler means of accounting for household size applied in previous studies is to multiply the projected number of households by the average per household car use. The projected number of households implicitly takes into account changes in average household size, since it is equal to the population size divided by average household size. Figure 4 shows that this approach yields the highest of all the projections, peaking about $20 \%$ higher than the benchmark case in 2030 . The result is driven by the fact that this method accounts for shifts in household size in the

\footnotetext{
${ }^{8}$ Alternatively, the effect can be explained by the fact that smaller households have larger per capita car use and therefore a compositional shift in the population toward smaller households leads to greater aggregate car use.
} 
demographic projection, but does not account for the fact that smaller households have lower car use; it applies constant car use per household throughout the projection.

When household composition, defined as number of adults versus children, is added to household size, projected car use increases by just a few percent. This relatively weak influence may be the result of two offsetting effects: more adult-only households, exerting upward pressure on car use rates, and an increasing share of single-parent households, exerting downward pressure on car use (cf. Figure 1d).

We conclude by applying a composition that differentiates between household size and age of household head combined (cf. Figure 1e). This projection yields results that are substantially different in both pattern and level from the projections accounting for each variable alone. Relative to the projection incorporating age alone, car use is lower by up to $7 \%$. The age-only projection does not account for the fact that the shift toward older households will also involve a shift toward smaller households with lower car use. Relative to the projection incorporating size alone, the projection incorporating age + size is higher through 2026 and lower thereafter. The size-only projection does not account for the baby boom-driven age effect which drives car use first higher, and then lower, than it otherwise would be. Adding gender of the household head in addition to the age of the household head and the size of the household yields slightly higher car use but does not effect the general shape of the projected car use pattern.

Taken together, these results imply that accounting for both age and size of households is warranted in projecting future car use. Adding gender of the householder and the adult/children composition of households has less effect. In addition, simple means of accounting for age and size such as using number of adults and number of households are insufficient to capture these demographic effects.

\section{Change in car use under different demographic compositions and alternative future demographic scenarios}

The extent to which a particular compositional variable affects future car use depends on the household projection employed. Under alternative assumptions about fertility, mortality, or union dissolution, the projected distribution of households by age, size, gender, and composition will change. As a result, the conclusions regarding the most important compositional variables to include in projected car use could also change.

To explore this possibility, we extend our analysis by investigating the sensitivity of projected car use to the alternative household projections presented in section $4 .{ }^{9}$

[Figure 5a-5e about here]

\footnotetext{
${ }^{9}$ Of course, changes in the cross-sectional pattern of car ownership and mean distance driven may have an equally important influence on projected car use. However since we lack information on changes in car use patterns across cohorts we restrict our analysis to the sensitivity of car use with respect to alternative household projection scenarios which can be constructed straightforward by assuming alternative future time paths of demographic parameters.
} 
We present our findings as follows. In the case of only one compositional variable we plot the change in projected car use relative to a projection based on population size alone (Figure 5a). If we have two or three compositional variables, we plot the ratio of the projection including both or all three variables to the projection including just one or two variables (Figure 5b, 5c, 5d and 5e). This approach controls for the differences in population size across scenarios with different demographic assumptions. Results can then be interpreted directly in terms of the importance of the compositional effect being tested, independently of the effect of differences in population size.

The results of Figure 5a imply that household age and size will be significant in all of the future demographic scenarios, since in all cases projected car use differs as compared to a projection based on population size alone. The effect of household size is smaller and not as sensitive to demographic conditions, leading to a 3-5\% increase in projected car use depending on the household scenario. The effect of household age is more pronounced, and more sensitive to the household scenario, peaking at $10-15 \%$ above the benchmark projection and ending at $-3 \%$ to $+12 \%$ in 2046 , depending on the demographic assumptions.

The results can also be used to examine the main causes of the sensitivity of car use to alternative assumptions. For example, the differences in car use between the high and low mortality scenario, after controlling for population size, are not very pronounced over the time period of the projection. Changes in mortality shift the distribution of households between middle- and older-aged categories (see Figure 3b). For example, lower mortality leads to a greater proportion in older households and a smaller proportion in middle-aged households, reducing overall car use since older households drive less. The differences in projected car use are small initially, since the increase in older households is concentrated in those households with driving patterns the most similar to the middle aged (i.e., the youngest households within the old-age group). Continued low mortality eventually leads to greater concentrations in the oldest households with the lowest level of driving. As a result, near the end of the projection period lower mortality is leading to an increasingly strong effect on total car use.

Differences in car use (controlled for population size) among the high and low fertility scenario are much more pronounced. Alternative fertility scenarios change the share of middle-aged households, and total car use is sensitive to this change. Lower fertility, for example, leads to a smaller share of young households, and a larger share of households in both the middle- and old-aged groups. The effect of the increase in middle-aged households (with high car use) dominates, and total car use increases. Projected changes in car use are even more pronounced if we assume alternative dissolution patterns, since these alternative scenarios lead to the largest shifts in the distribution of households by age (Figure 3b). For example, higher dissolution rates shift the distribution of households toward the middle-aged group, which has relatively high car use, leading to an increase in overall car use. 
In Figure $5 b$ and $5 c$ we consider the effect of adding a second compositional variable to either the age of the household head or the size of the household. We plot projected car use relative to projections that account for age of household head or household size only. Results confirm conclusions reached in the previous section regarding the relative importance of different compositional variables. Adding sex to age (Figure 5b) results in relatively small changes in car use, although in the low dissolution case the effect is the largest, reaching $4 \%$ by the end of the projection period. Lower dissolution rates lead to a larger share of male-headed households, which have higher car use than female-headed households. However, this result does not include the size effects associated with changing dissolution rates, which would act in the opposite direction. Adding size to age has a pronounced effect in all scenarios, although it is considerably lessened in the low dissolution scenario (and considerably increased in the high dissolution scenario).

Adding composition (by adults vs. children) to size (Figure 5c) has a relatively small effect in all scenarios while adding age to size has a substantial effect in all cases.

We conclude by considering three compositional variables: age and sex of household head together with household size (Figure 5d and 5e). Adding gender of the household head (in addition to age and size of the household) does not change the pattern of future car use and this is independent of the future demographic scenario we assume (Figure 5d). Compared to Figure 5b, part of the gender specific effect has already been taken up by the compositional variable household size such that by adding gender changes in car use across alternative future demographic scenarios are very small. The importance to distinguish by household size (in addition to age and sex) is verified again in Figure 5e. However, compared to Figure $5 b$, the effect of adding size across alternative future demographic scenarios is smaller if gender has already been considered in addition to age.

Our results confirm the robustness of our initial conclusion that household age and size are important compositional variables to include in projections of future car use. By adding gender to a composition by age and size (Figure 5d), not much additional change in car use can be observed. We may therefore conclude that age and size are indeed the most appropriate compositional variables within our set of household characteristics that we consider. With respect to the alternative future demographic scenarios our results indicate that the quantitative relevance to a specific demographic composition may change under alternative demographic future scenarios while the qualitative shape persists.

\section{Conclusions}

Demand patterns for transportation with private vehicles are closely connected to demographic variables, including those reflective of life-cycle stages. We find, as have previous studies, that demand for household transportation varies significantly by different subgroups of the population defined by household characteristics such as age and gender of the householder, size, and age composition. By combining cross-sectional variations in travel behavior by demographic characteristics with a new projection of 
households in Austria, we illustrate that future compositional changes in the population by living arrangements could substantially influence demand for transportation.

Furthermore, we show that projections are sensitive to the particular type of demographic disaggregation employed. These results suggest that demographic disaggregation not only has the potential to improve forecasts of future travel demand, but also to emphasize the importance of careful choice of variables by which to disaggregate the population.

Demographic changes could be important for at least two reasons in addition to those analyzed here. First, we assume that category-specific car ownership and use rates remain constant. If, however, these rates changed differentially across categories, the effect of compositional changes on aggregate demand could be either exacerbated or dampened. Second, one of the reasons why category-specific rates might be expected to change is the likely existence of cohort effects (a demographic variable). For example, as baby boom women age, they are likely to increase the rate of car ownership in elderly age groups.

\section{[Figure 6 about here]}

Whether our results indicate that compositional changes could have a substantial influence on future travel behavior needs to be judged relative to the influence of other factors, including behavioral and technological changes. Referring to data provided by the Austrian environmental ministry (Figure 6), vehicle kilometers traveled (VKT) per adult is forecasted to increase by about $62 \%$ during the period 1996 to 2030 compared to an increase of $155 \%$ over a historical period of similar length from 1967 to $1996 .{ }^{10}$ At the same time, changes in energy efficiency and transportation fuels could lead to an improvement in CO2 emissions per vehicle kilometer of 40\% over the period 1996 to 2030 compared to an improvement of only 15\% for the historical period 1967 to 1996.

Compared to these projected changes in VKT and technological factors, our predicted changes in car use resulting from compositional changes are modest. Taking the projection by household age, sex and size as an example and considering the medium variant of the household projections, differences from the projection which ignore composition (the constant per capita projection) do not exceed 8\%. For an application forecasting aggregate transportation energy use 50 years into the future, an $8 \%$ adjustment is relatively small given the scope for changes driven by behavioral or technological change. On the other hand, the projection with composition shows a different dynamic which may be important, with demand peaking earlier and then declining, in sharp contrast to the constant per capita projection and the projections presented in Figure 6. In addition, the difference between the two projections is nearly $8 \%$ in the short term (2010-2015). Over this shorter time horizon, an $8 \%$ absolute difference in projected demand is likely to be much more important in judging the difficulty of meeting greenhouse gas emission reduction targets, or for planning for changes in demand for road capacity, for example.

\footnotetext{
${ }^{10}$ We would like to thank Günther Lichtblau (Austrian environmental ministry) and Alexander Hanika (Statistic Austria) who provided the data on VKT, energy efficiency and the Austrian adult population.
} 


\section{References}

Bongaarts, J. (1987) The projection of family composition over the life course with family status life-tables. In John Bongaarts, Thomas K. Burch, and Kenneth W. Wachter (eds.) Family demography: Methods and their application, edited by International Studies in Demography, 189-212, New York: Oxford University Press.

Buettner, T. and Grubler, A. (1995) The birth of a "green" generation?:Generational dynamics of consumption patterns. Technological Forecasting and Social Change 50, 113-134.

Carlsson-Kanyama, A. and Linden, A.-L. (1999) Travel patterns and environmental effects now and in the future: Implications of differences in energy consumption among socio-economic groups. Ecologcial Economics 30, 405-417.

Dahl, C.A. and Sterner, T. (1991) Analysing gasoline demand elasticities: A survey. Energy Economics 13(3), 203-210.

Doblhammer, G., Lutz, W. and Pfeiffer, C. (1997) Tabellenband und Zusammenfassung erster Ergebnisse: Familien- und Fertilitätssurvey (FFS) 1996. Österreichisches Institut für Familienforschung, Vienna, Austria.

Grenning, L.A. and Jeng, T.H. (1994) Lifecycle analysis of gasoline expenditure patterns. Energy Economics 16(3), 217-228.

Greening, L.A. et al. (1997) Prediction of household levels of greenhouse gas emissions from personal automobile transportation. Energy 22 (5), 449-460.

Hammel, E.A., Hutchinson, D. W., Wachter, K.W., Lundy, R.T. and Deuel, R.Z. (1976) The SOCSIM Demographic-Sociological Microsimulation Program, Berkeley, Institute of International Studies, University of California (Research Series No. 27).

Hanika, A. (1996) Volkszählung 1991: Paritäts-Fruchtbarkeitstafeln, Statistische Nachrichten 6/1996, 426-431.

Hanika, A. (1999) Realisierte Kinderzahl und zusätzlicher Kinderwunsch: Ergebnisse des Mikrozensus 1996, Statistische Nachrichten 5/1999, 311-318.

Hanika, A. (2000) Bevölkerungsvorausschätzung 2000-2050 für Österreich und die Bundesländer, Statistische Nachrichten 12/2000, Statistik Austria, 977ff.

Kayser, H.A. (2000). Gasoline demand and car choice: Estimating gasoline demand using household information. Energy Economics. 22(3), 331-348. 
Lutz, W. (ed., with ass. eds.: Baguant, J., Prinz, C., Toth, F.L. and Wils, A.B.) (1994), Population - Development - Environment: Understanding their Interactions in Mauritius, Berlin, Springer-Verlag.

U.S. National Research Council (NRC) (1997) Toward a Sustainable Future: Addressing the long-term effects of motor vehicle transportation on climate and ecology. Special Report 251. National Academy Press, Washington, DC, USA.

O'Neill, B.C. and Chen, B. S. (2002) Demographic determinants of household energy use in the United States, in W. Lutz, A. Prskawetz and W.C. Sanderson (eds.) Population and Environment: Methods of Analysis, A Supplement to Vol. 28 of Population and Development Review, New York, Population Council, 53-88.

Prskawetz, A., Vikat, A., Philipov, D. and Engelhardt, H. (2002) Pathways to Stepfamily Formation in Europe: Results from the FFS. Mimeo, Max Planck Institute for Demographic Research.

Pucher, J., Evans, T. and Wenger, J. (1998) Socioeconomics of urban travel: Evidence from the 1995 NPTS. Transportation Quarterly 52(3), 15-33.

Schipper, L., Bartlett, S., Hawk, D. and Vine, E. (1989) Linking lifestyles and energy use: A matter of time? Annual Review of Energy and the Environment 14, p. 273.

Spain, D. (1997) Societal trends: The aging baby boom and women's increased independence. Report prepared for the U.S. Dept. of Transporation, Order no. DTFH6197-P-00314.

Statistic Austria (1998) Energieverbrauch der Haushalte 1996/97: Ergebnisse des Mikrozensus Juni 1997, Vienna, Austria.

UN ECE PAU. FFS model questionnaire for women. Available at http://www.unece.org/ead/pau/ffs/

van Imhoff, E. and Keilman, N. (1991), Lipro 2.0: An Application of a Dynamic Demographic Projection Model to Household Structure in The Netherlands, Amsterdam/Lisse and Berwyn PA, Swets \& Zeitlinger Inc.

Zeng Yi (1991), Family Dynamics in China: A Life Table Analysis, Wisconsin, The University of Wisconsin Press.

Zeng, Yi, Vaupel, J. and Wang, Z. (1997) A Multidimensional model for projecting family households with an illustrative numerical application. Mathematical Population Studies, Vol. 6, No. 3, 187-216. 
Zeng Yi, Vaupel, J. and Wang, Z. (1999), Household projection using conventional demographic data, in W. Lutz, J.W. Vaupel and D.A. Ahlburg (eds.), Frontiers of Population Forecasting, A Supplement to Volume 24 of Population and Development Review, New York, Population Council, 59-87. 


\section{Appendix A: Methodology of household projection}

ProFamy is a software package for a multi-dimensional, macro-dynamic model for projecting households and population. In the ProFamy model, the individual is chosen as the basic unit of the projection. The individuals in the starting population are derived from a census or survey. Individuals in the projected population are classified according to 8 dimensions of demographic status: age, sex, marital status, parity, number of children living at home, co-residence with parents, household type (private or collective), and area of residence (rural or urban). Characteristics of the reference person (or household "markers") are applied to derive the distributions by household type and size. Because the model deals with two sexes, children and parents, a harmonic-mean procedure is used to ensure consistency between females and males, and children and parents. At the end of the projection, the numbers of non-familial members who co-reside with the reference persons is estimated indirectly (Zeng et al., 1997).

\section{Baseline population and status identification}

The 1996 Austrian micro-census, which includes a sample of the Austrian population of the order of 64,183 individuals ${ }^{11}$, provides the baseline population for our household and population projection. In order to adjust this sample to get the correct total population size by age, sex, and marital status, we need to provide ProFamy with information on the total population in the starting year. The $100 \%$ tabulation of the total population $(8,054,423)$ classified by single year of age, sex and marital status is derived by applying the population weights provided in the 1996 micro-census. Table A.1 summarizes the status of individuals as used in our projections.

[Table A.1 about here]

This information, as derived from the 1996 micro-census, serves as the input for BasePop, the sub-program of ProFamy, in order to produce the base population for projections in ProFamy.

\section{$\underline{\text { Standard schedules }}$}

The basic structure of the demographic accounting equation in ProFamy is as follows:

number of persons of age $x+1$ with status $i$ at time $t+1=$

(number of persons of age $x$ with status $i$ at time $t$ ) +

(number of entries into status $i$ which occur in the year $(t, t+1)$ among persons of age $x+1$ at time $t+1)-$

(number of exits out of status $i$ which occur in the year $(t, t+1)$ among persons of age $x$ at time $t$ ).

\footnotetext{
${ }^{11}$ It should be noted that, there are some individual cases in the micro-census data set containing no information required. Those cases with missing value are excluded from the baseline population. Consequently, 57,030 out of 64,183 are used in the final sample for ProFamy.
} 
The number of demographic events in year $(t, t+1)$ and at ages $x$ or $x+1$ is calculated as the number of persons aged $x$ and at risk of occurrence of the event in the year, multiplied by the projective probability of occurrence of an event that leads to a status transition in the year $(t, t+1)$ and at age $x$ or $x+1$ in completed years, fitting in a period-cohort observational plan for analysis and projection. ${ }^{12}$ It is obvious that the standard schedules defined by age-, sex-, (sometimes parity-, and marital-status-) specific probabilities (or rates) ${ }^{13}$ are extremely important for the projection results.

To derive the standard schedules for the various events we use three sources:

The 1996-1997 Austrian Fertility and Family Survey (FFS) contains detailed information on partnership formation and dissolution, childbearing, leaving parental home, migration, and other events, based on a retrospective investigation of 6120 individuals aged 20-54 during the period December 1995 up to May 1996. Applying the method of survival analysis, we derive the following standard schedules from the FFS data:

(1) Probability of leaving parental home

(2) Transition probability from single to married

(3) Transition probability from single to cohabiting

(4) Transition probability from cohabiting to married

(5) Transition probability from cohabiting to single

(6) Transition probability from married to divorced

(7) Transition probability from divorced to cohabiting

Given the small sample size, the FFS data did not allow for the construction of standard schedules for the transition probability from widowed to cohabiting. For the latter transition probability, we therefore assume that it is equal to the transition probability from divorced to cohabiting. A further shortcoming of the FFS data set is the fact that it only includes individuals up to age 54. It is un-reasonable to assume that those aged above 54 do not get divorced. To solve this problem we used 1996 age- and sex-specific numbers of divorces from Statistic Austria. Combining these numbers with the number of married couples derived from the 1996 micro-census data set, we calculated the age- and sex-specific divorce rate, and used it in constructing the standard schedule of divorce rates. ProFamy software will automatically calculate the standard schedule for the transition from married to widowed based on the sex-specific mortality schedules and differences in age at marriage between men and women.

\footnotetext{
${ }^{12}$ Below, when we use the term probability, we always mean probability according to this definition. In this approach, the demographic transitions under study are assumed to depend on some (but not all) of the other statuses at the beginning or the middle of a one-year interval (e.g., giving birth depends on age, parity and marital status of the mother, but does not depend on co-residence with parents). The adoption of the computational strategy in the model, which assumes that births occur throughout the first half and the second half of a year, but other events occur in the middle of the year, will significantly decrease the biases caused by not considering the interfering events while projecting the occurrences of an event.

${ }^{13}$ With regard to the input data, the ProFamy software package allows the standard schedules to be either occurrence/exposure rates or probabilities. If one chooses to use occurrence/exposure rates, the ProFamy model will automatically transform all rates into probabilities. In our case, we transformed all standard schedules, except those for the frequencies of migration, from occurrence/exposure rates into probabilities, before we used them as input into the model.
} 
Although the FFS data set contains information on fertility, the small sample size again did not permit use of these data to derive standard schedules for fertility. We use the 1996 micro-census data set, which provides detailed information on birth biographies, to construct standard schedules for parity- and age- specific fertility schedules.

(1) Single women fertility

(2) Cohabiting women fertility

(3) Divorced women fertility

(4) Married women fertility

It is noteworthy that we set 15 as the starting age of giving birth, and parity 5 as the highest parity. Since the number of births by widowed women is too small in the microcensus we assumed widowed women will not give any birth in our projection. Similarly, the number of births of parity 4 and 5 by single and cohabiting women, and of parity 5 by divorced women are very small and we neglect these births for our projections.

The following standard schedules are provided by Statistic Austria:

(1) Probability of survival

(2) Transition probability from divorced to married

(3) Transition probability from widowed to married

(4) Frequency distribution of immigration by age and sex

(5) Frequency distribution of emigration by age and sex

\section{$\underline{\text { Summary measure }}$}

Base year

While standard schedules offer information on the status transition of the future population characterized by age, sex, and marital status; summary measures provide the assumption of the total amount of all transitions in the future years. All these assumptions should be based on the information from the base year and also start from the base year.

Basically, we obtained information for the summary measures of the base year from the three sources of deriving standard schedules mentioned above.

(1) From the FFS we calculated the proportion of those who eventually leave the parental home, the propensity of first marriages, the mean age at $1^{\text {st }}$ marriage, the propensity from married to divorced, the propensity from single to cohabiting, the propensity from cohabiting to married, the propensity from cohabiting to single, and the propensity from divorced to cohabiting.

(2) From the 1996 micro-census data we derived the ratio of the non-married women fertility to the married women fertility.

(3) From Statistic Austria we obtained figures relating to life expectancy at birth, total fertility rate, number of emigrants and immigrants, sex ratio at birth, and propensity for the widowed and divorced to remarry. Since we lack proper data to estimate the propensity of widowed to cohabiting, we assume that it is the same as the propensity for the widowed to remarry. 
The total fertility rate by parity derived from the micro-census data is the result of the combination of different cohorts. It is not adequate to use it to represent the distribution of total fertility rate by parities. Therefore, we obtained data on total fertility by parities of the latest cohorts of Austrian women born in 1946-1950 who have experienced childbearing (Hanika, 1996), and use it to represent the distribution of the total fertility rate by parities. Moreover, we assume no births given by widowed women.

Numbers for external migrants are obtained from Statistic Austria for the year 1998. We assume that there is no significant change in terms of external migrants between 1996 and 1998 and apply these numbers to our base population of 1996.

From the base population, ProFamy automatically calculates the proportion of persons living in collective household by age and sex among those who are not living with children or parents plus the average number of other relatives and non-relatives of different households by size.

\section{Future changes}

For women's' mean age at giving birth, the number of external migration, the total fertility rate and the life expectancy at birth we apply the assumptions as suggested in the latest projections of Statistic Austria (Hanika, 2000).

The mean age at giving birth is assumed to increase ( following a logistic curve) from 28.14 years in 1996 to 30 years in 2030 and it is kept constant at 30 years from 2030 onwards till 2046.

The external migration (Table A.2) is derived from the population projections by Statistic Austria.

[Table A.2 about here]

For total fertility rate three assumptions are stated:

(1) The medium variant assumption assumes total fertility rate will increase ( following a logistic curve) from 1.42 in 1996 to 1.5 by the year 2020, and will maintain at a level of 1.5 till the year 2046 .

(2) The low variant assumption assumes total fertility rate will linearly decrease to 1.2 by the year 2020 and remain constant thereafter.

(3) The high variant assumption assumes total fertility rate will linearly increase to 1.8 by the year 2020, and remain constant thereafter.

For life expectancy at birth, three further assumptions are stated:

(1) The medium variant assumes an increase that follows a logistic curve: from 74.7 to 80 years for males and from 80.9 to 85.5 years for females in the period between 1996 and 
2030. For the period between 2030 and 2050, life expectancy is assumed to increase from 80 to 82.5 years for males and from 85.5 to 87 years for females.

(2) The low variant assumes a linear increase from 74.7 to 78.0 years for males and 80.9 to 84 years for females in the period between 1996 to 2030 . From 2030 onwards, life expectancy is assumed to stay constant for both sexes.

(3) The high variant assumes that life expectancy at birth between 1996 and 2030 will increase linearly to 82 years for males and 87 years for females and increase linearly up to 86 years for males and 90 years for females until 2050 .

For the union dissolution rate, we construct a low and high union dissolution scenario assuming that Austria follows the Italian (low union dissolution scenario) or alternatively the Swedish pattern (high union dissolution scenario) of union dissolution by the year 2046 (between 1996 and 2046 we apply a linear interpolation).

According to the 1990 round of the FFS Survey, out of 19 European countries, Swedish women of birth cohort 1952-59 had the highest proportion of union dissolution by age 35 (0.35); about 1.5 times that of their Austrian counterparts (0.23). Conversely, Italian women of the same birth cohort had the lowest proportion of union dissolution by age 35 (0.05); about 0.26 times of that of their Austrian counterparts. Thus we form three scenarios for the whole life proportion of union dissolution by married or cohabiting men and women, by assuming proportionate changes to the base year of whole life proportions of union dissolution by married and cohabiting men and women derived from the 1995/1996 FFS data. These are: 0.37 for married women, 0.38 for married men, 0.20 for cohabiting women and 0.29 for the cohabiting men.

(1) The medium scenario assumes the whole life proportion of union dissolution by married and cohabited women and men will remain constant at the 1996 level till the end of the year 2046 of the projection.

(2) The high scenario of union dissolution assumes the proportion of union dissolution between the year 1996 and 2046 will linearly increase from 0.37 to 0.56 for married women and from 0.38 to 0.58 for married men, while the proportion will linearly increase from 0.20 to 0.31 for cohabiting women and from 0.29 to 0.45 for cohabiting men in the same period.

(3) The low scenario of union dissolution assumes the proportion of union dissolution will linearly decrease to 0.09 for both the married men and women by year 2046. It further assumes a decrease to 0.05 for the cohabiting women and 0.07 for the cohabiting men by year 2046 .

All other summary measures are assumed to be constant in the future. By applying this set of assumptions, we actually assume little changes in the behavior of household formation and dissolution except that changes in women's mean age at giving birth and total fertility rate will to a certain degree affect the timing and volume of household expansion by childbearing. 


\section{Appendix B: Determination of cohabiting status}

We use information on the marital status variable together with the following information to determine cohabitation status:

(1) household number - people cohabiting are coded in the same household

(2) age- only adults aged above 15 are considered for cohabitation. The age difference of those cohabiting is set within a 10-year range and this may underestimate the prevalence of cohabitation to a certain extent.

(3) marital status

(4) gender- only opposite gender cohabitation is considered

(5) position in the household- household head or respective relationships to the household head

(6) a variable (b5kz1) which indicates partnership relationship of household members while cohabiting partners are stated as other persons of household heads

Consequently, 8 types of cohabitation for two adults of different gender and living in the same household are derived:

(1) one is head, one is spouse; one is married, one is non-married

(2) one is head, one is spouse; both are non-married

(3) one is head, one is other person who states to be a partner of the household head; no spouse is present in the household

(4) one is coded as child, one is coded as "other person"; one is married, one is nonmarried; age difference within a range of 10 years; with or without information on the variable "b5kz1", given that "b5kz1" is not completely coded

(5) one is coded as child, one is "other person"; both are non-married; age difference within a range of 10 years; with or without information on the variable "b5kz1"

(6) both are coded as "parents", and at least one is un-married

(7) one is parent, one is other person, at least one is un-married, with information provided by "b5kz1"

(8) two other persons, at least one is unmarried, with information on the variable b5kz1. 


\section{Tables:}

Table 1: ANOVA analysis applied to distance traveled for alternative compositional variables

\begin{tabular}{|c|c|c|c|c|c|c|}
\hline & Sum of squares & Df & Mean Square & F-statisic & Significance & $\begin{array}{r}\% \text { of Total } \\
\text { Variance }\end{array}$ \\
\hline \multicolumn{7}{|c|}{ Age of household head } \\
\hline Between groups & $1.30 \mathrm{E}+13$ & 12 & $1.10 \mathrm{E}+12$ & 7531.628 & 0 & 4.2 \\
\hline Within groups & $3.00 \mathrm{E}+14$ & 2027985 & $1.50 \mathrm{E}+08$ & & & \\
\hline \multicolumn{7}{|c|}{ Age and sex of household head } \\
\hline Between groups & $1.80 \mathrm{E}+13$ & 25 & $7.30 \mathrm{E}+11$ & 5003.61 & 0 & 5.8 \\
\hline Within groups & $2.90 \mathrm{E}+14$ & 2027972 & $1.50 \mathrm{E}+08$ & & & \\
\hline \multicolumn{7}{|l|}{ Size of household } \\
\hline Between groups & $1.40 \mathrm{E}+13$ & 6 & $2.40 \mathrm{E}+12$ & 16424.32 & 0 & 4.5 \\
\hline Within groups & $3.00 \mathrm{E}+14$ & 2027991 & $1.50 \mathrm{E}+08$ & & & \\
\hline \multicolumn{7}{|c|}{ Number of adults and children in the household } \\
\hline Between groups & $1.90 \mathrm{E}+13$ & 28 & $6.90 \mathrm{E}+11$ & 4773.032 & 0 & 6.1 \\
\hline Within groups & $2.90 \mathrm{E}+14$ & 2027969 & $1.40 \mathrm{E}+08$ & & & \\
\hline \multicolumn{7}{|c|}{ Age of household head and size of household } \\
\hline Between groups & $2.80 \mathrm{E}+13$ & 68 & $4.10 \mathrm{E}+11$ & 2948.789 & 0 & 9.0 \\
\hline Within groups & $2.80 \mathrm{E}+14$ & 2027929 & $1.40 \mathrm{E}+08$ & & & \\
\hline Total & $3.10 \mathrm{E}+14$ & 2027997 & & & & \\
\hline
\end{tabular}


Table 2: Assumptions on future changes of summary measures

\begin{tabular}{|l|l|r|r|r|}
\hline \multicolumn{1}{|c|}{} & & 1996 & 2020 & 2046 \\
\hline Fertility & TFR & 1.34 & 1.50 & 1.50 \\
\cline { 2 - 5 } & $1^{\text {st }}$ birth & 0.55 & 0.61 & 0.61 \\
& $2^{\text {nd }}$ birth & 0.39 & 0.43 & 0.43 \\
& $3^{\text {rd }}$ birth & 0.21 & 0.23 & 0.23 \\
& $4^{\text {th }}$ birth & 0.11 & 0.12 & 0.12 \\
& $5^{\text {th }}$ birth & 0.00 & 0.10 & 0.10 \\
\hline Life expectancy & Female & 80.90 & 84.0 & 86.7 \\
& Male & 74.70 & 78.3 & 81.6 \\
\hline Mean age at childbearing & 28.14 & 30.00 & 30.00 \\
\hline Immigration & Female & 33,793 & 37,174 & 37,174 \\
& Male & 38,930 & 42,826 & 42,826 \\
\hline Emigration & Female & 27,736 & 26,667 & 24,729 \\
& Male & 36,536 & 35,128 & 32,574 \\
\hline
\end{tabular}


Table 3: Assumptions on future fertility and mortality levels

\begin{tabular}{|l|c|c|c|}
\hline & TFR & Male & Female \\
\hline Low & & 78 & 84 \\
Medium & 1.2 & 82.5 & 87 \\
High & 1.5 & 86 & 90 \\
\hline
\end{tabular}


Table A.1: Individual demographic status identification ${ }^{14}$

\begin{tabular}{ll}
\hline Demographic status & Definition \\
\hline Age x & by single year of age, 0 to 95 (the highest age) \\
Sex s & 1 female; 2 male \\
Marital status m & 1 single; 2 married; 3 widowed; 4 divorced; \\
& 5 cohabiting \\
Co-residence with parents k & 1 with two parents; 2 with one parent; \\
& 3 with no parents; 4 living in collective household \\
Parity p & 0 to 5 (the highest parity) \\
Co-residence with children c & 0 to 5 (the highest parity) \\
Household type & 1 private household; 2 collective household \\
\hline
\end{tabular}

${ }^{14}$ Residence area (rural/urban) could be used as a further individual status identification variable.

${ }^{15}$ Given that the status of cohabiting is not coded in the micro-census statistics of June 1996, we had to determine this status ourselves (see Appendix B). 
Table A.2: Immigration and Emigration numbers for 1998-2050

\begin{tabular}{|c|cc|c|cc|c|cc|}
\hline year & immigrants & emigrants & year & immigrants & emigrants & year & immigrants & emigrants \\
\hline 1998 & 72,723 & 64,272 & 2016 & 79,716 & 62,894 & 2034 & 80,000 & 58,858 \\
1999 & 86,710 & 66,923 & 2017 & 79,818 & 62,638 & 2035 & 80,000 & 58,717 \\
2000 & 77,242 & 65,510 & 2018 & 79,895 & 62,363 & 2036 & 80,000 & 58,555 \\
2001 & 77,280 & 65,311 & 2019 & 79,956 & 62,071 & 2037 & 80,000 & 58,427 \\
2002 & 77,327 & 65,136 & 2020 & 80,000 & 61,795 & 2038 & 80,000 & 58,283 \\
2003 & 77,388 & 64,915 & 2021 & 80,000 & 61,533 & 2039 & 80,000 & 58,156 \\
2004 & 77,468 & 64,741 & 2022 & 80,000 & 61,286 & 2040 & 80,000 & 58,043 \\
2005 & 77,572 & 64,551 & 2023 & 80,000 & 61,039 & 2041 & 80,000 & 57,913 \\
2006 & 77,705 & 64,385 & 2024 & 80,000 & 60,797 & 2042 & 80,000 & 57,803 \\
2007 & 77,867 & 64,220 & 2025 & 80,000 & 60,561 & 2043 & 80,000 & 57,669 \\
2008 & 78,063 & 64,078 & 2026 & 80,000 & 60,327 & 2044 & 80,000 & 57,549 \\
2009 & 78,289 & 63,949 & 2027 & 80,000 & 60,109 & 2045 & 80,000 & 57,420 \\
2010 & 78,531 & 63,839 & 2028 & 80,000 & 59,887 & 2046 & 80,000 & 57,303 \\
2011 & 78,779 & 63,714 & 2029 & 80,000 & 59,719 & 2047 & 80,000 & 57,156 \\
2012 & 79,019 & 63,590 & 2030 & 80,000 & 59,508 & 2048 & 80,000 & 57,035 \\
2013 & 79,240 & 63,469 & 2031 & 80,000 & 59,349 & 2049 & 80,000 & 56,899 \\
2014 & 79,430 & 63,305 & 2032 & 80,000 & 59,168 & 2050 & 80,000 & 56,767 \\
2015 & 79,590 & 63,108 & 2033 & 80,000 & 59,018 & & & \\
\hline
\end{tabular}




\section{Figures:}

Figure 1a: Mean distance driven and car ownership by age of household head, 1997

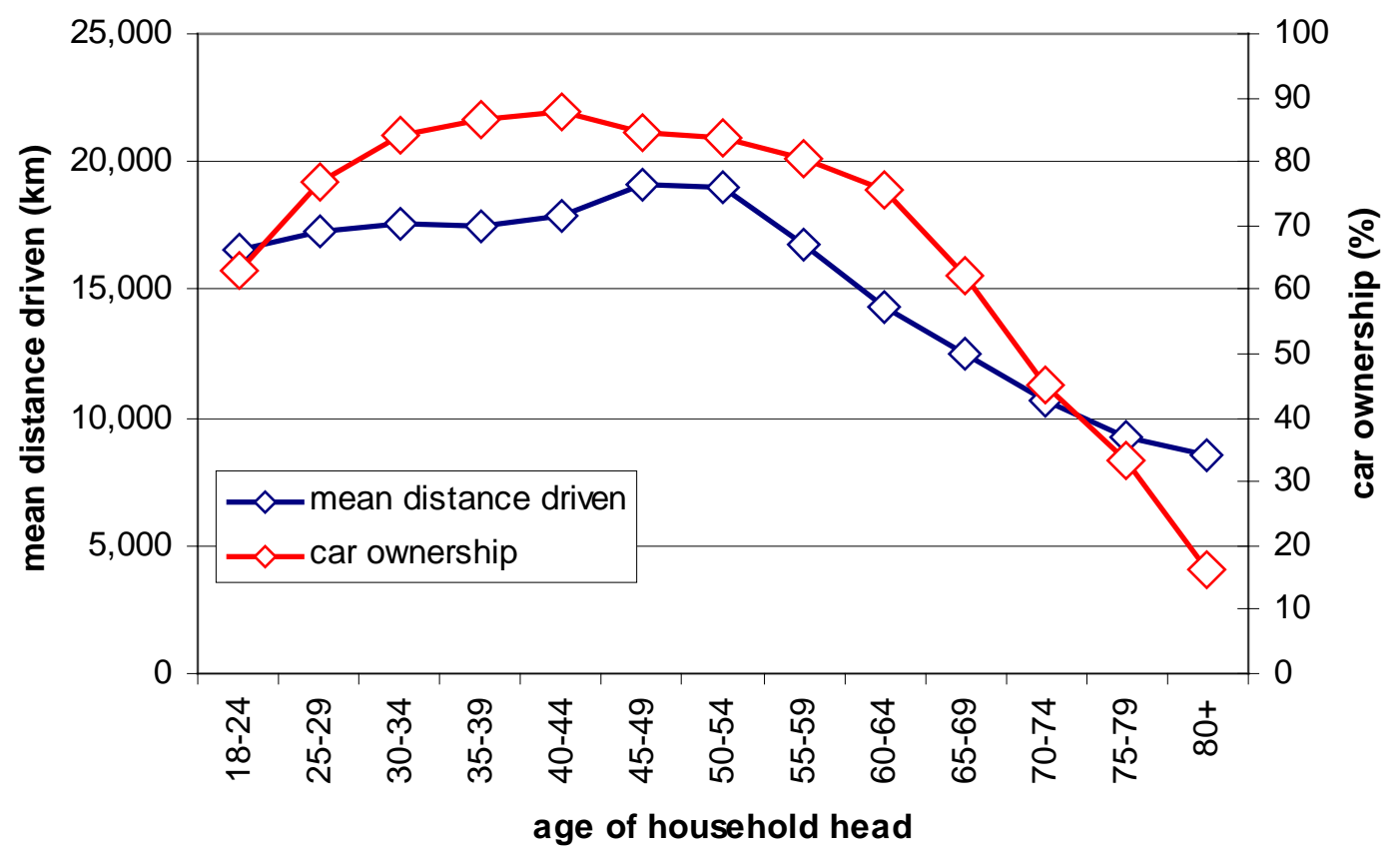

Figure 1b: Mean distance driven and car ownership by age and sex of household head, 1997

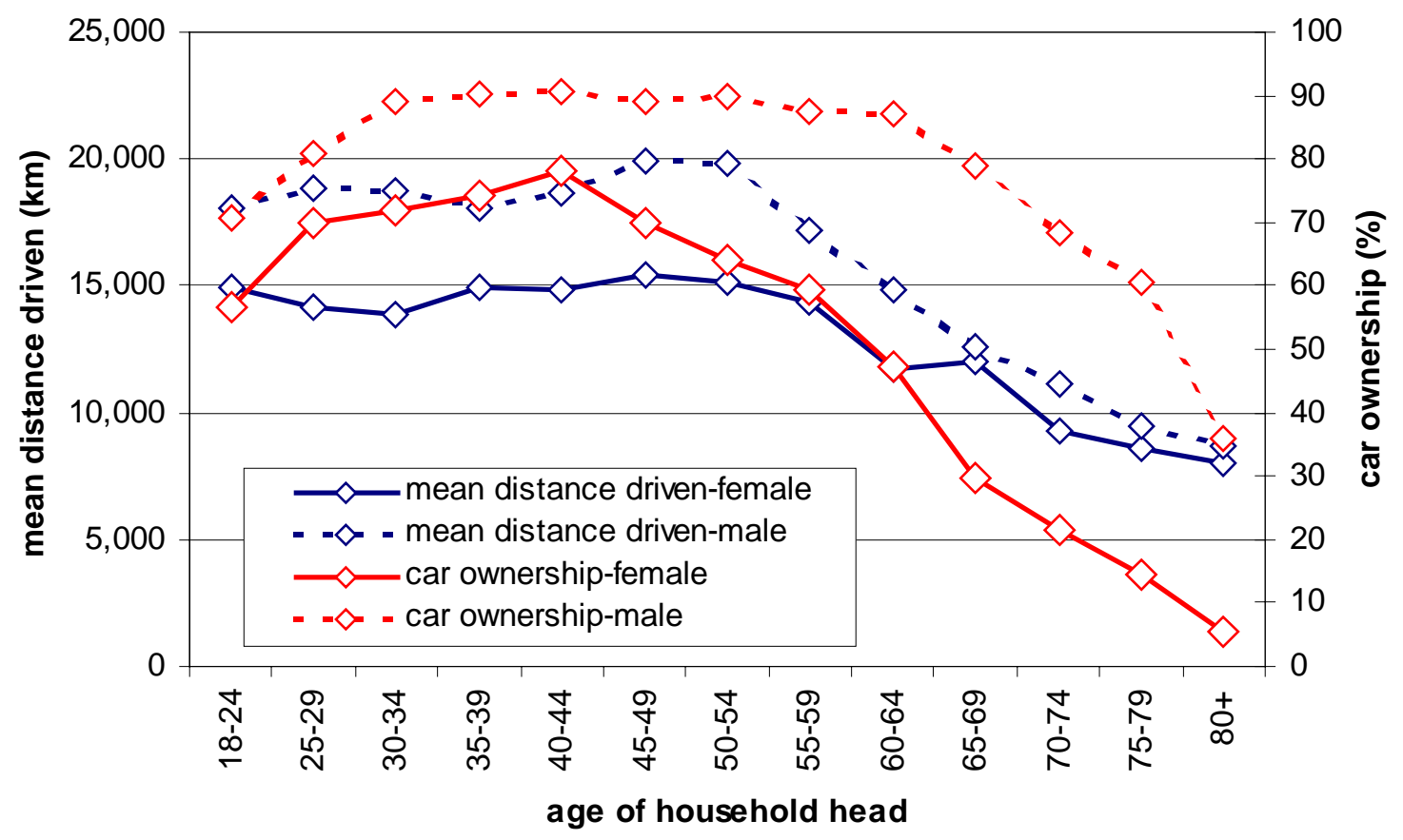


Figure 1c: Mean distance driven and car ownership by household size, 1997

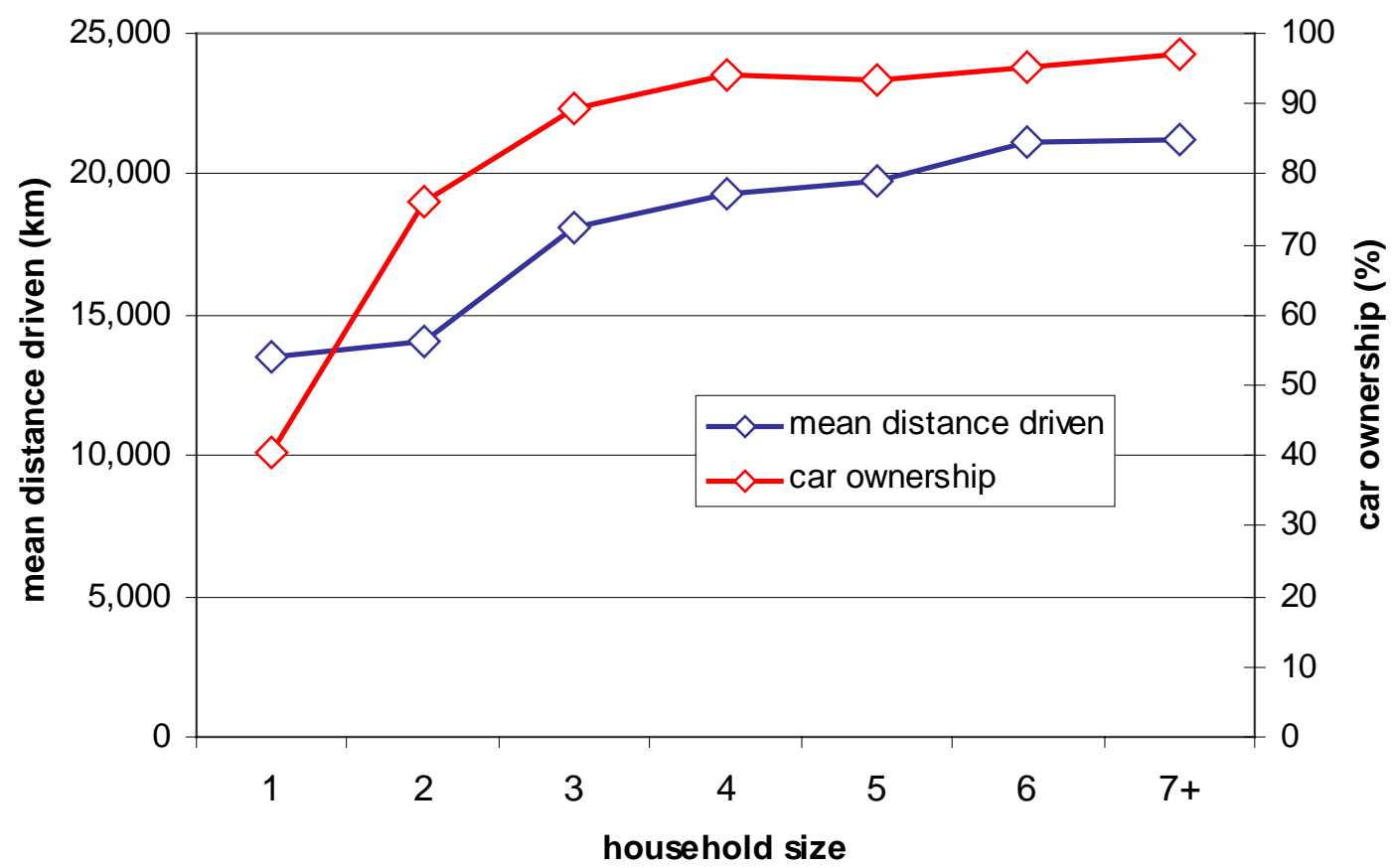

Figure 1d: Mean distance driven and car ownership by household size and number of children, 1997
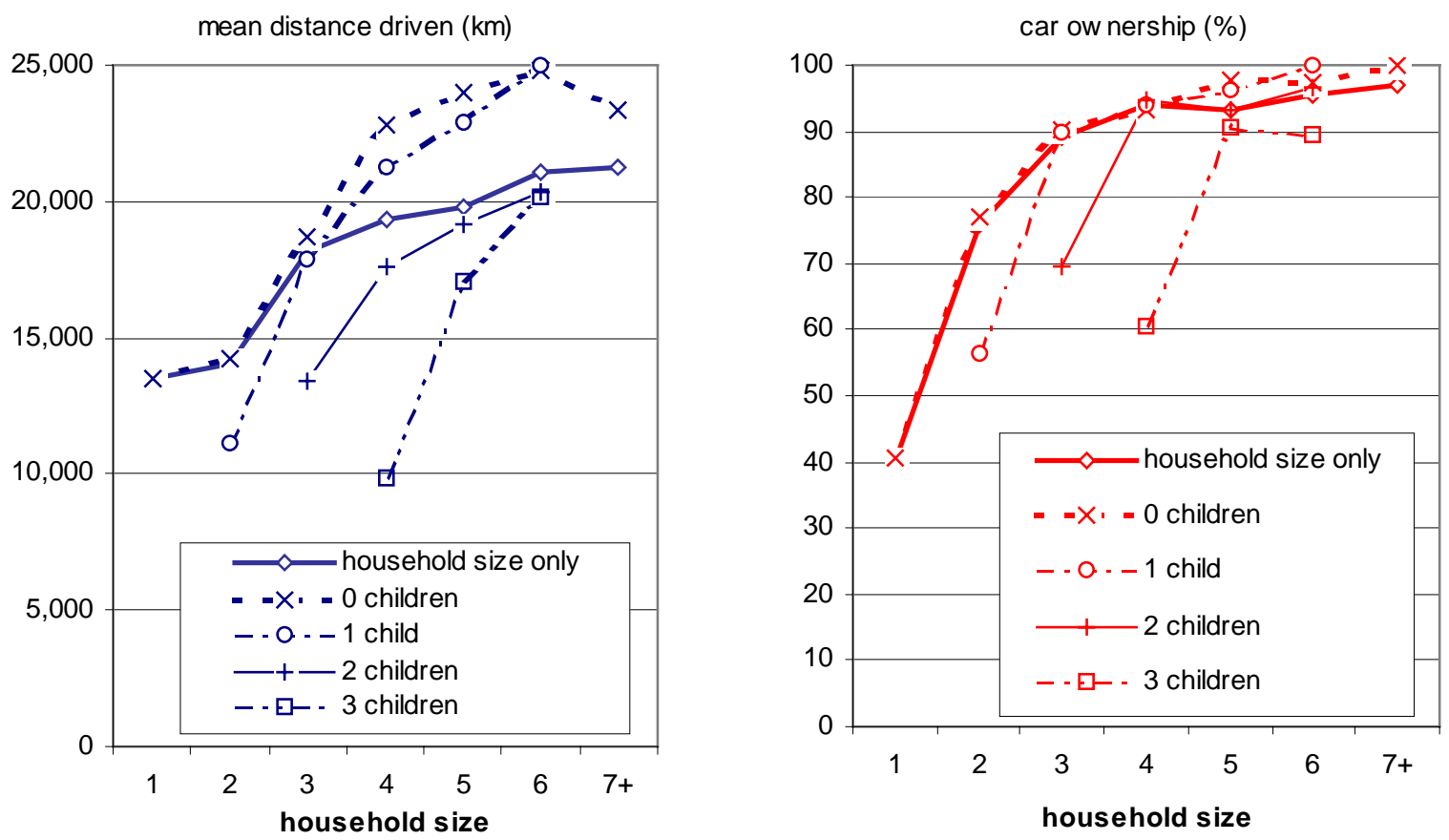
Figure 1e: Mean distance driven and car ownership by age of household head and household size, 1997
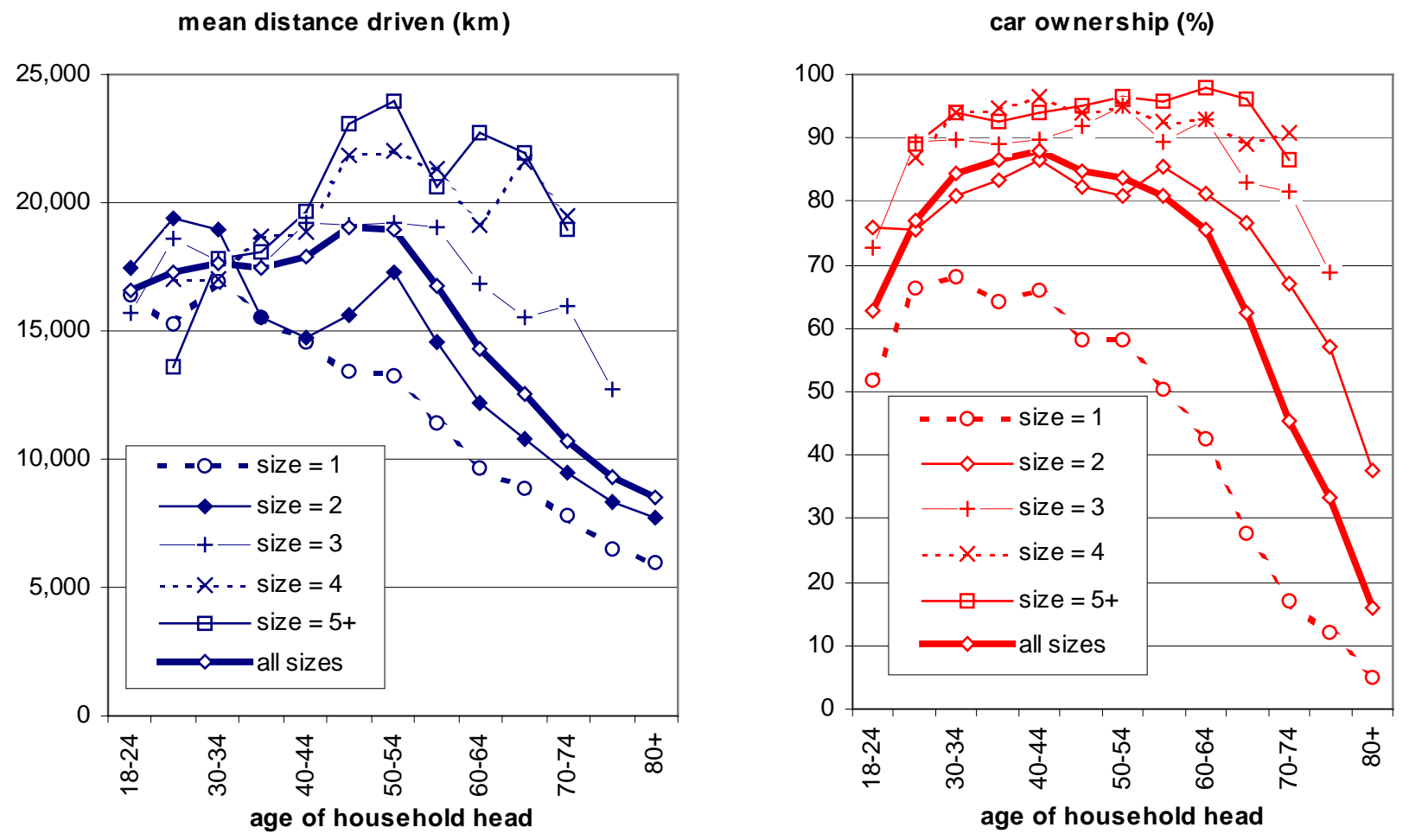
Figure 2a: Projected population size, number of adults and number of households

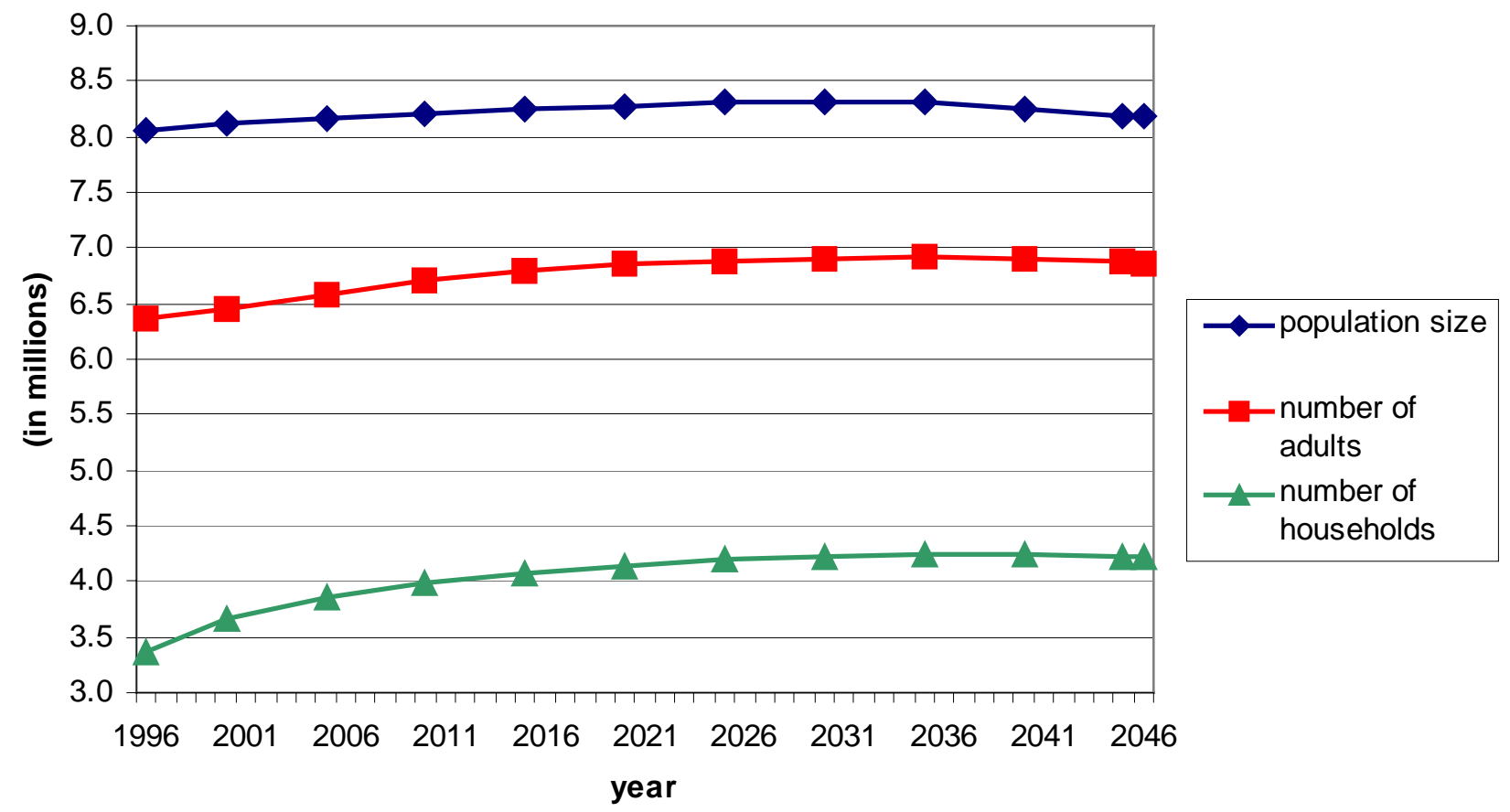

Figure 2b: Projected age structure of the population

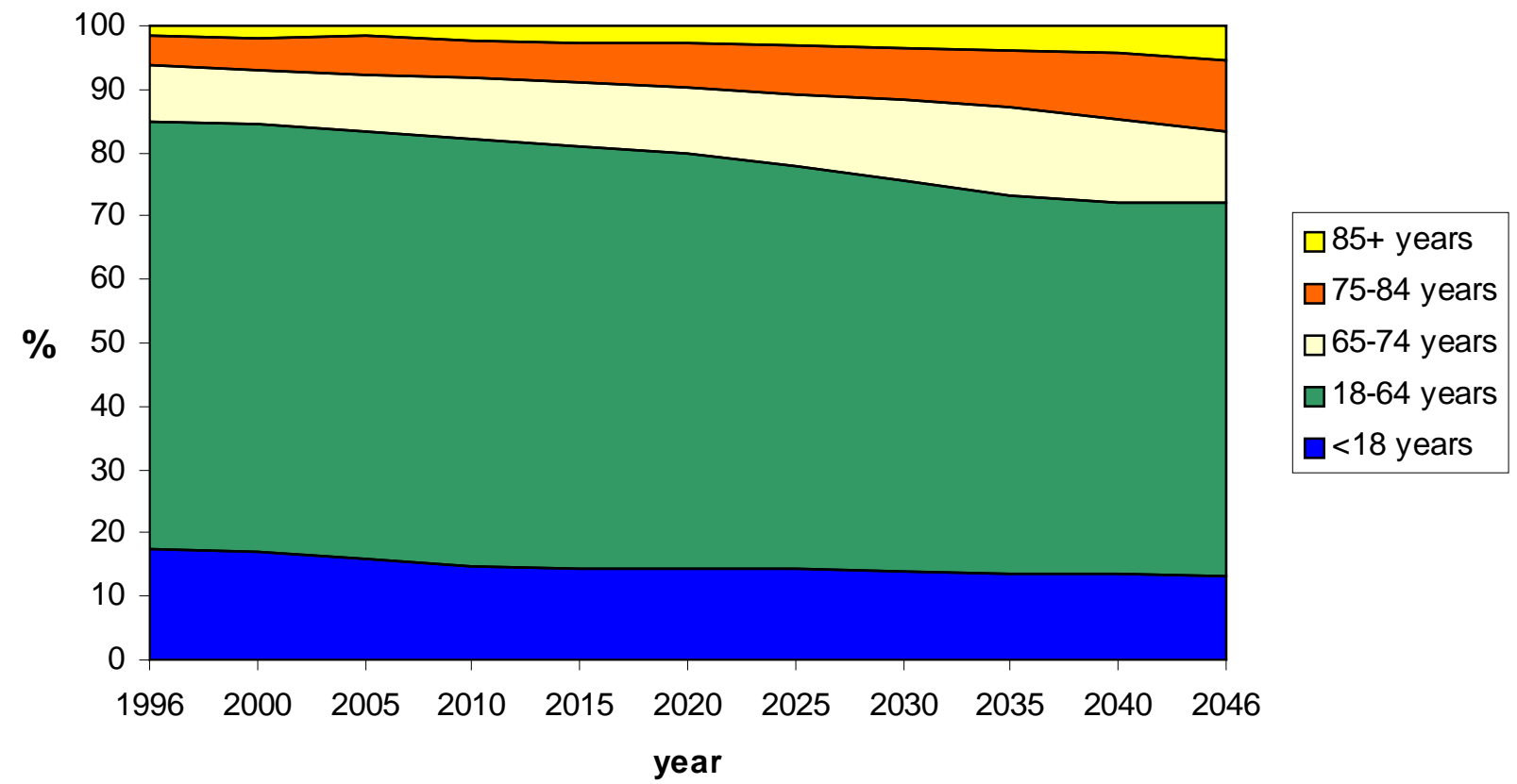


Figure 2c: Projected number of households by age of household head

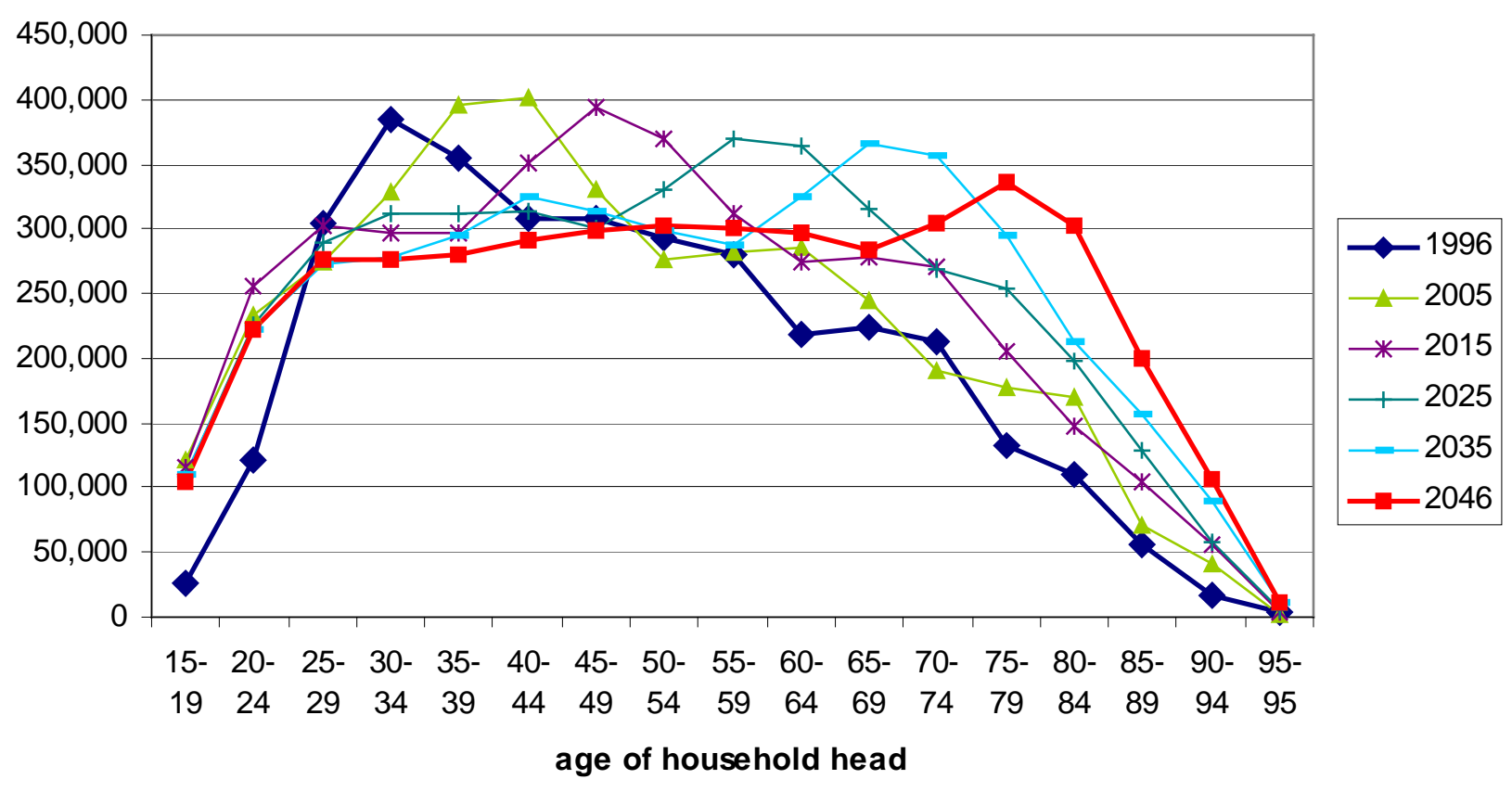

Figure 2d: Projected number of male headed households by age of household head

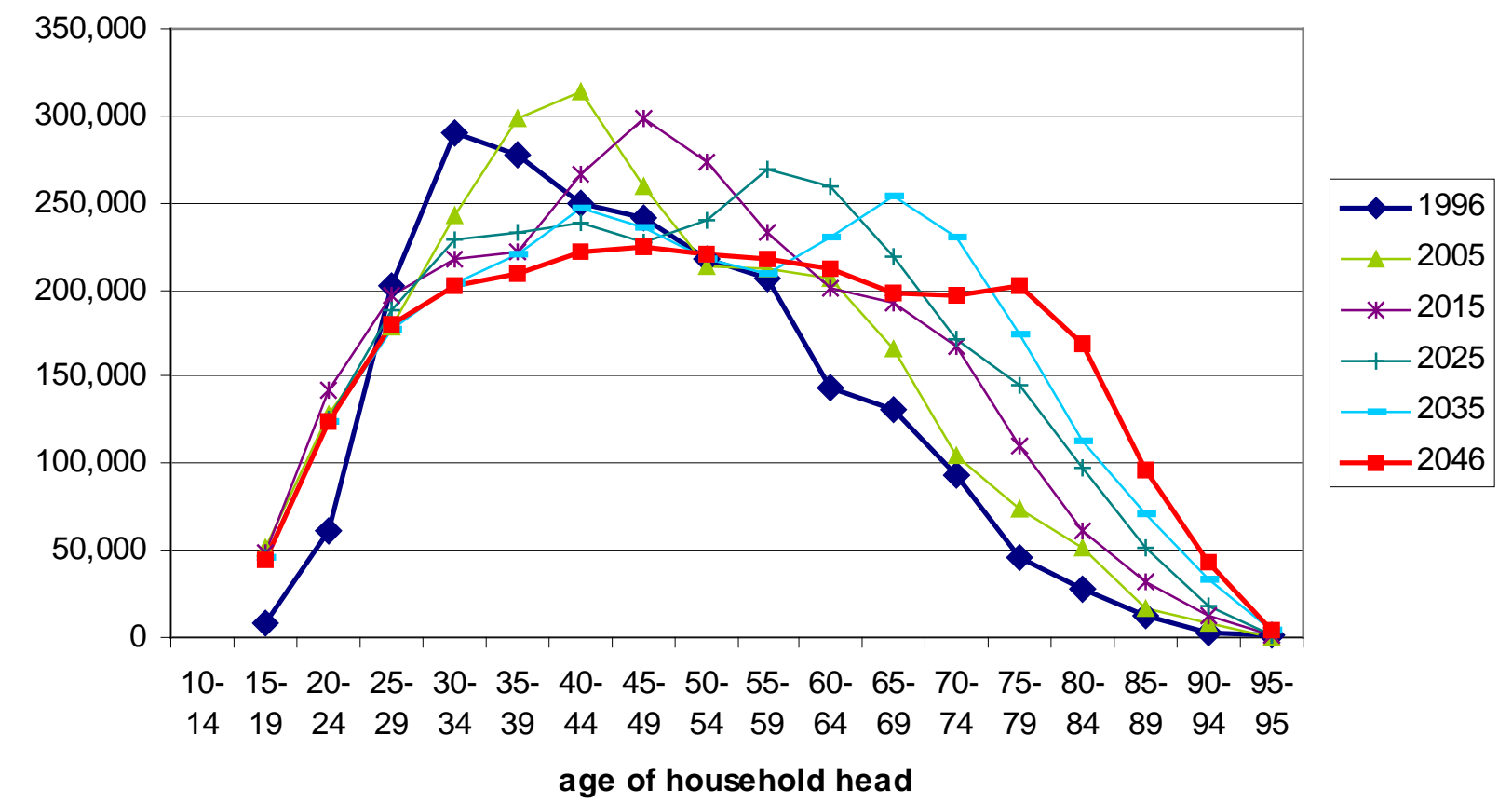


Figure 2e: Projected number of female headed households by age of household head

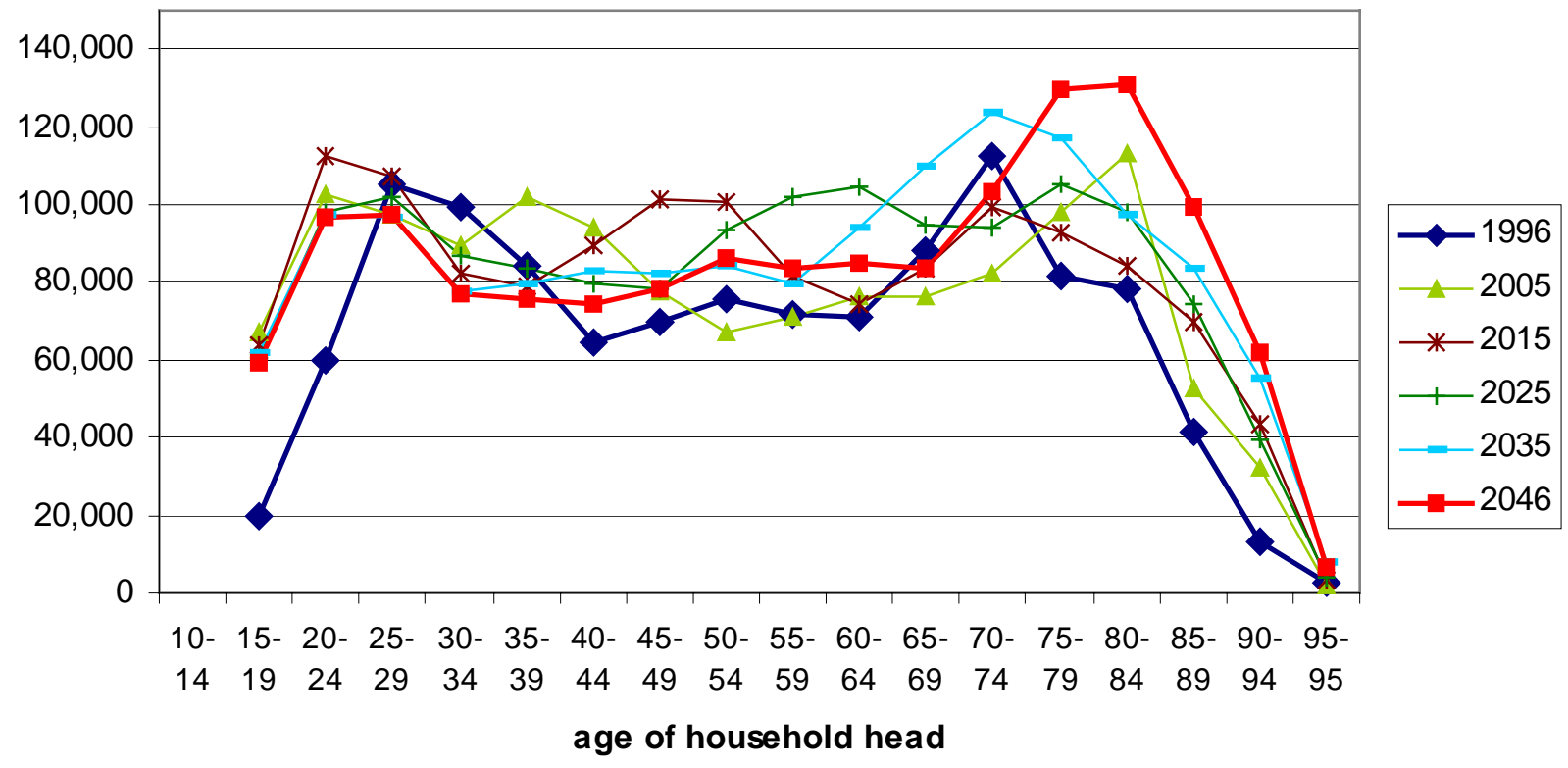

Figure 2f: Projected number of households by household size

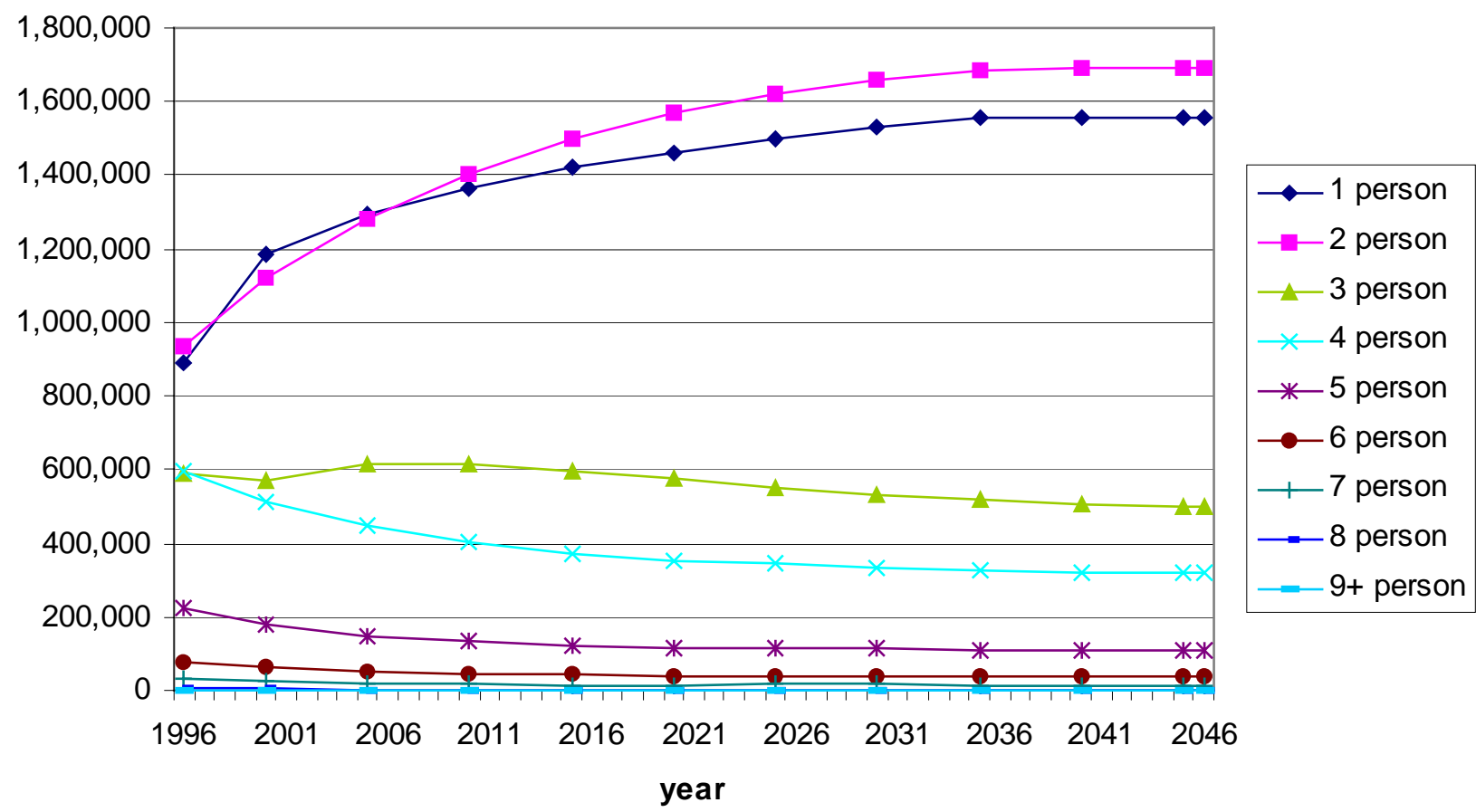


Figure 2g: Projected number of households by number of adults and children

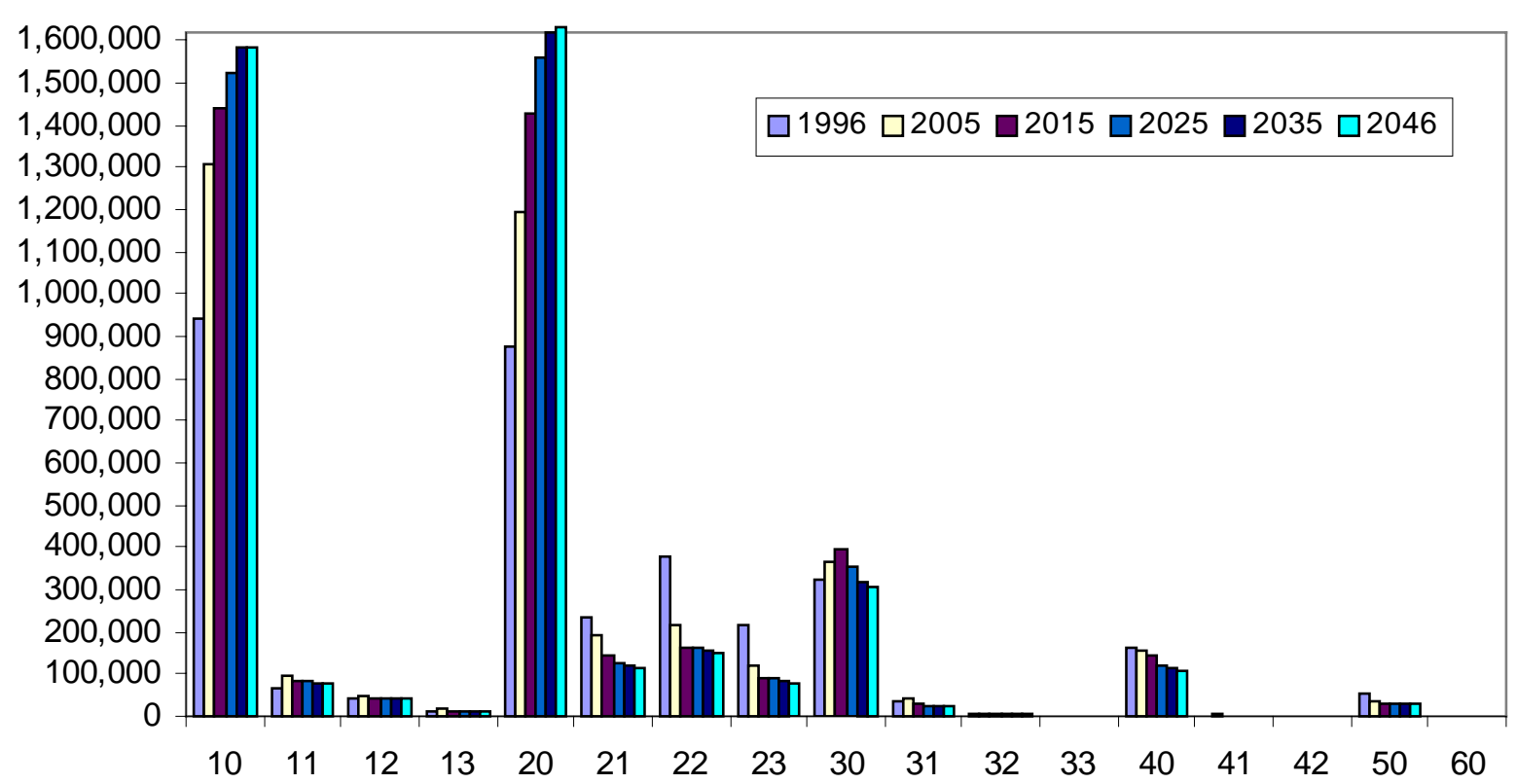

house hold categories (first digit $=$ number of adults; second digit $=$ number of children) 
Figure 3a: Projection of population size, number of adults and number of households under alternative future demographic scenarios
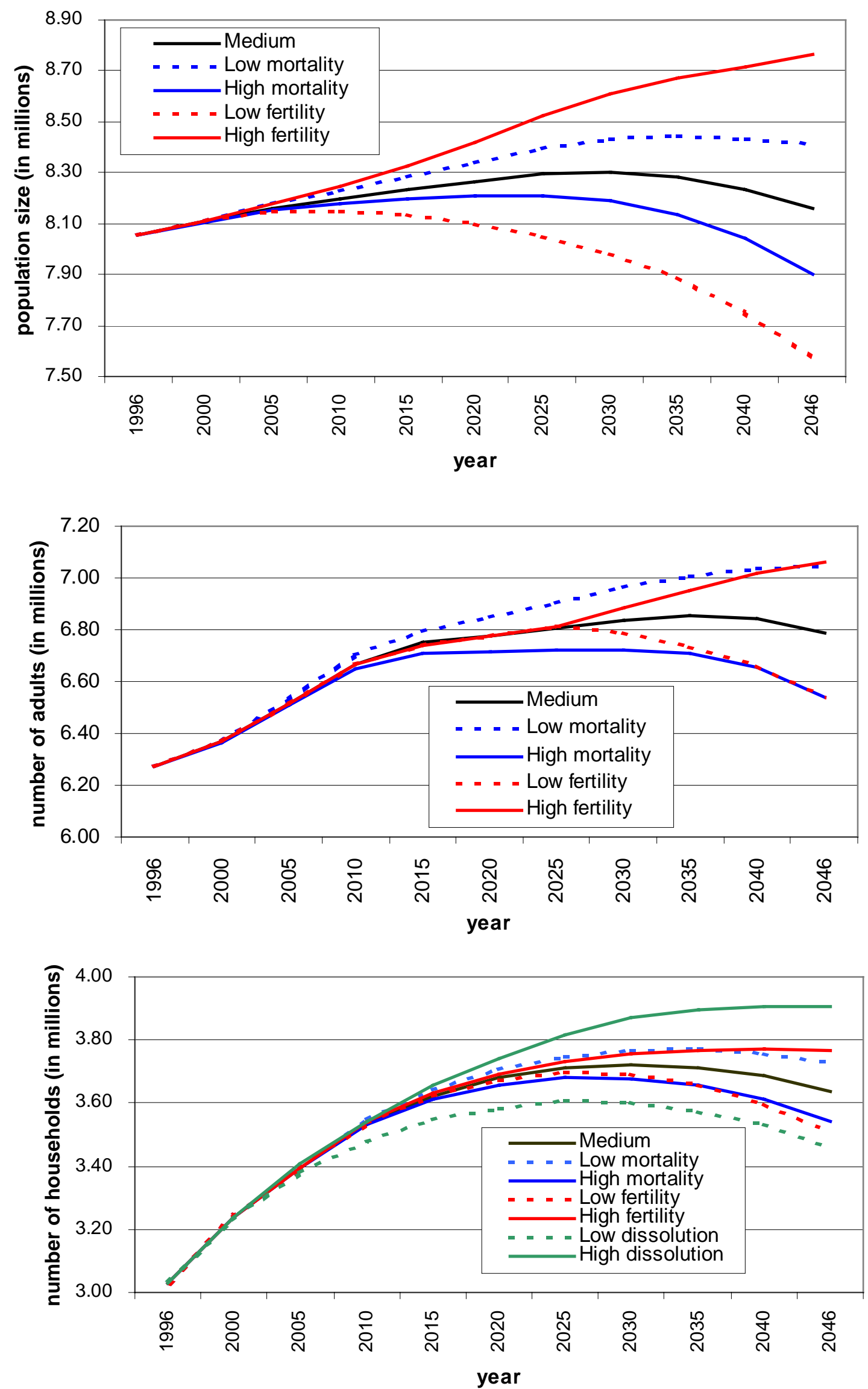
Figure 3b: Projection of the share of 15-29, 30-59 and 60+ years old household heads under alternative future demographic scenarios
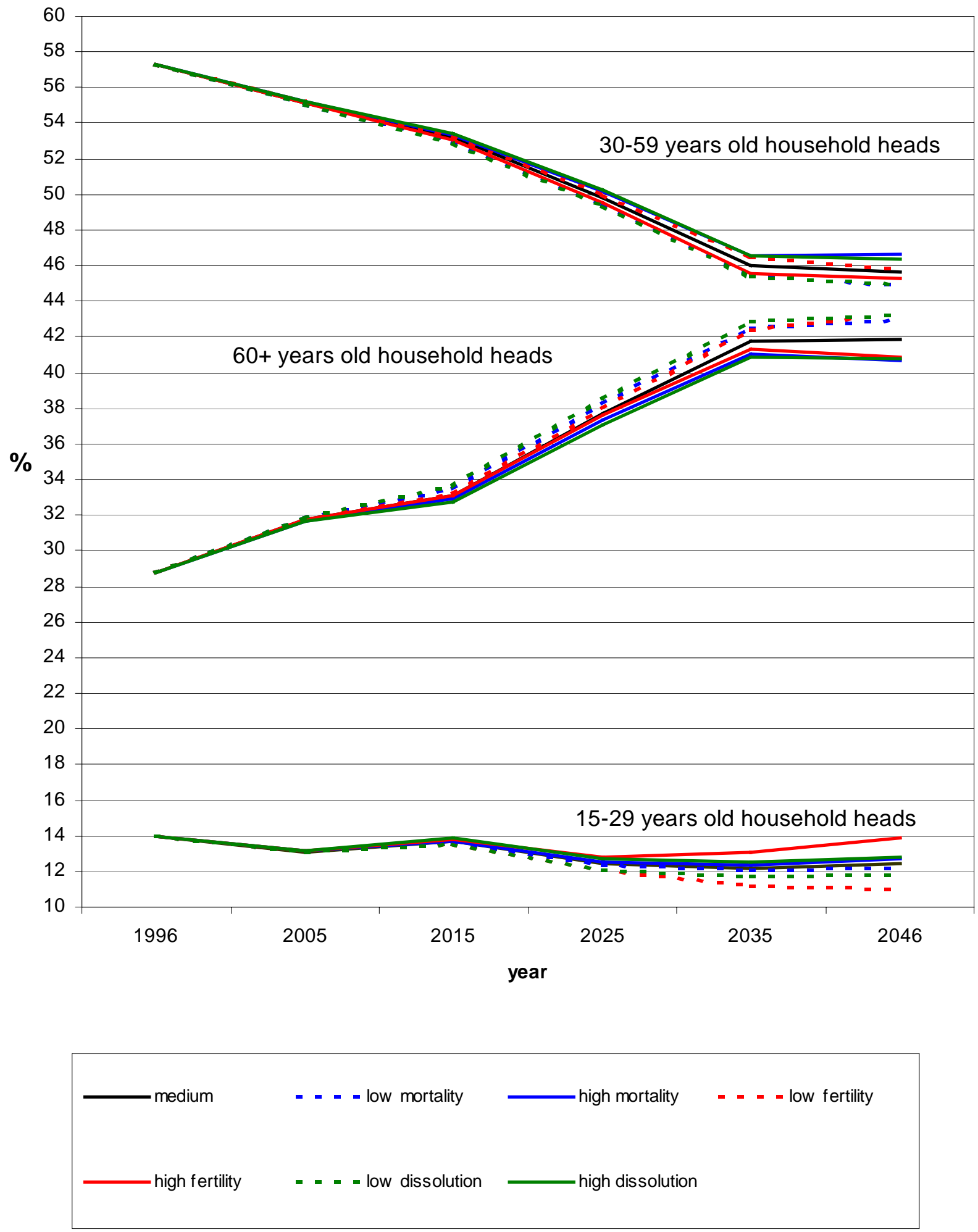
Figure 3c: Projection of the share of one, two and 3+ person households under alternative future demographic scenarios
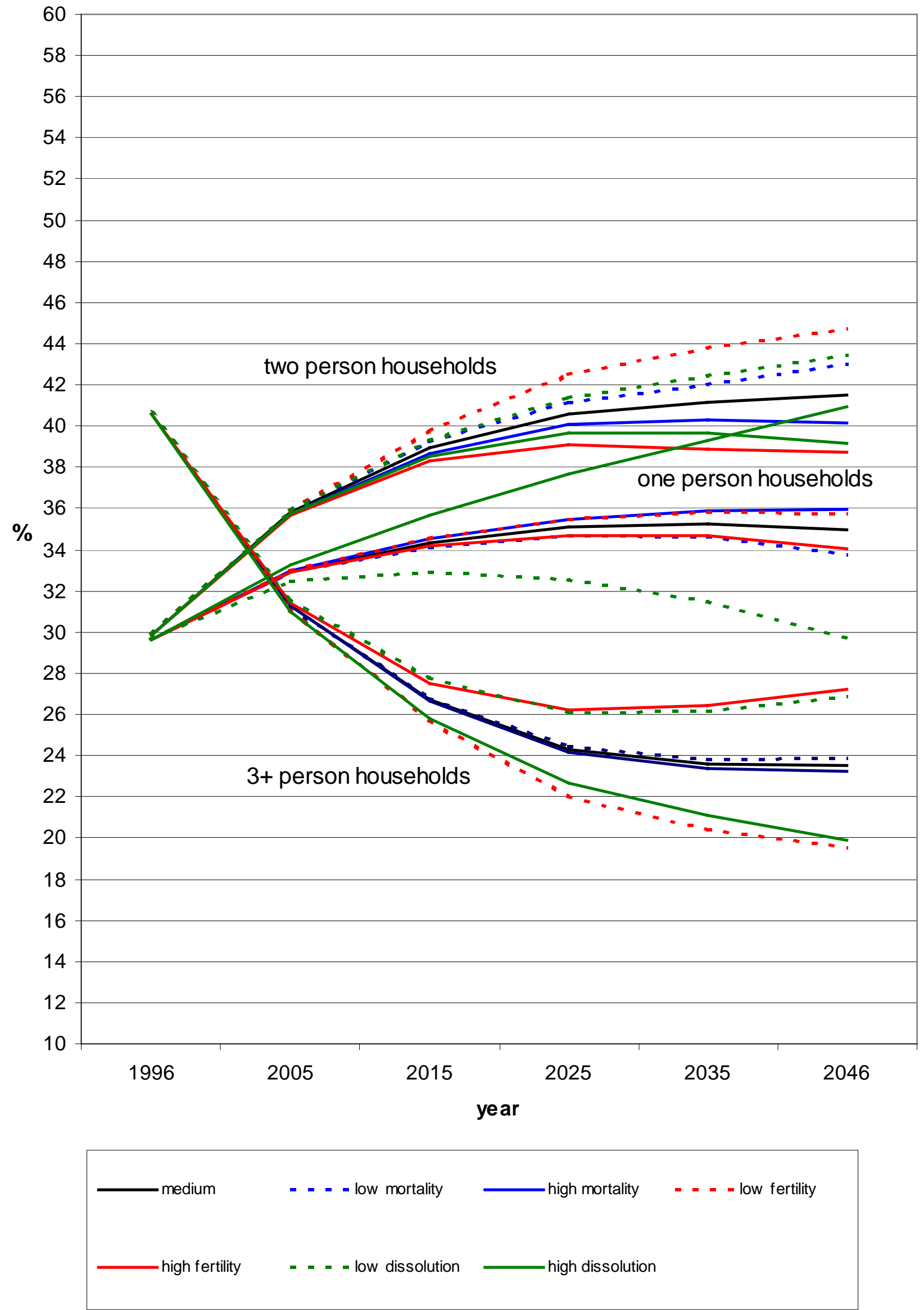
Figure 4: Change in car use under different demographic compositions; medium variant of the household projections

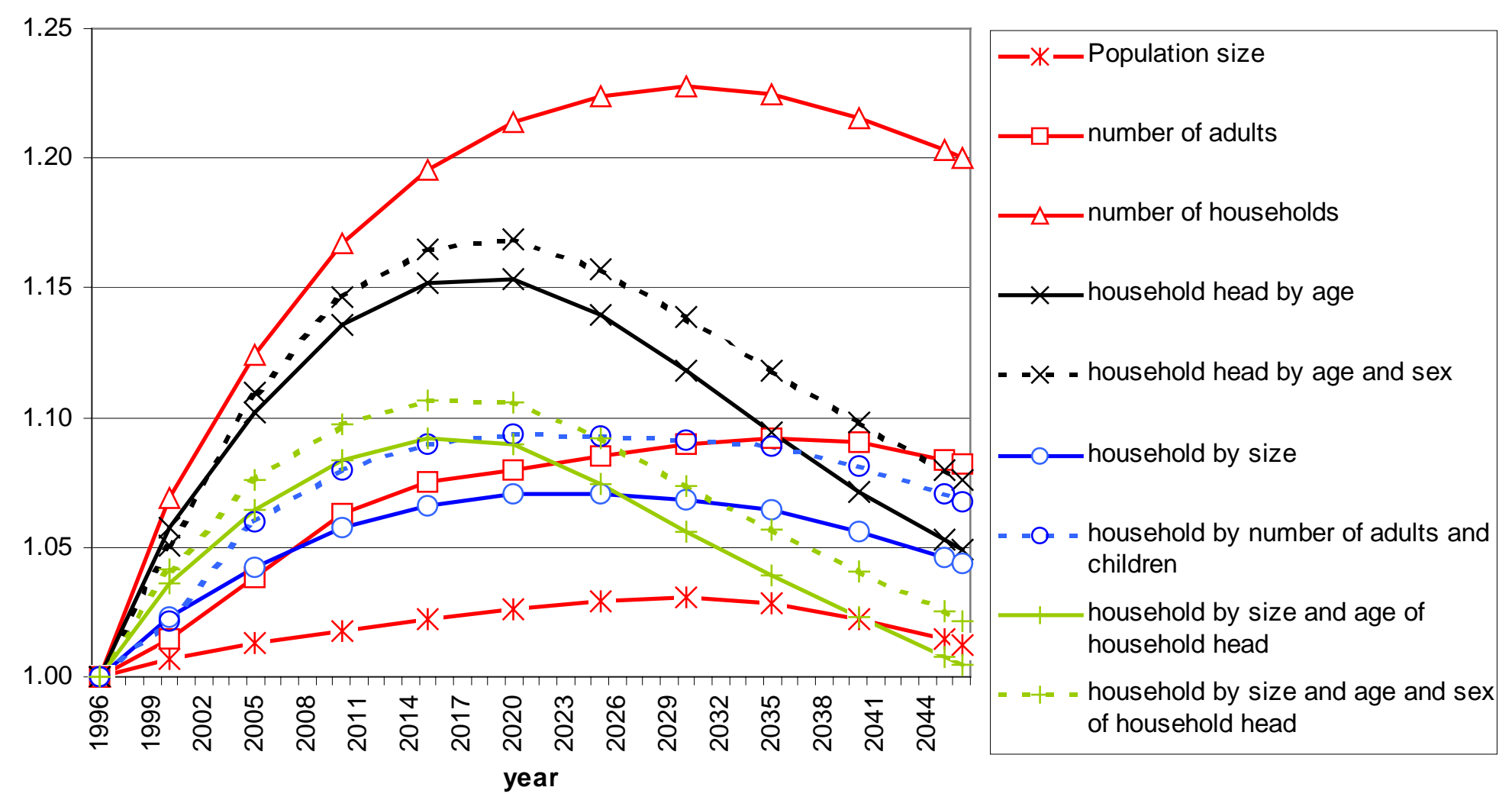


5a: Change in car use for alternative demographic scenarios by size of household and age of household head relative to a projection by population size

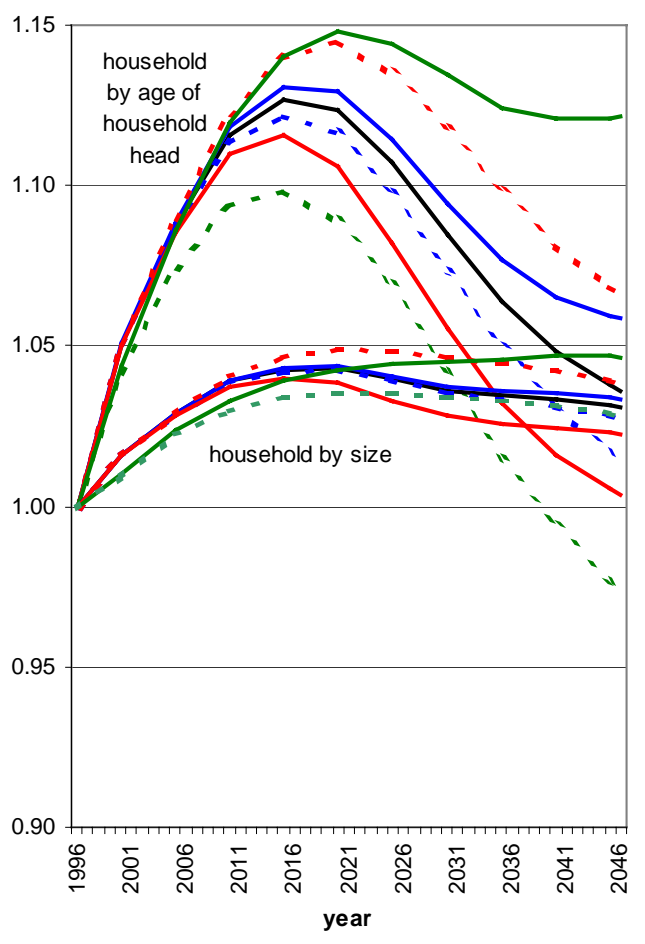

$$
\begin{array}{|ll|}
\hline \text { medium } & \text { high mortality }_{\text {high fertility }}^{\text {high dissolution }} \\
\text { - - - low mortality } & \ldots \text { - - low fertility } \\
\text { - - - low dissolution } & \\
\hline
\end{array}
$$

5b: Change in car use for alternative demographic scenarios by age and sex of household head and by household size and age of household head relative to a projection by age of household head

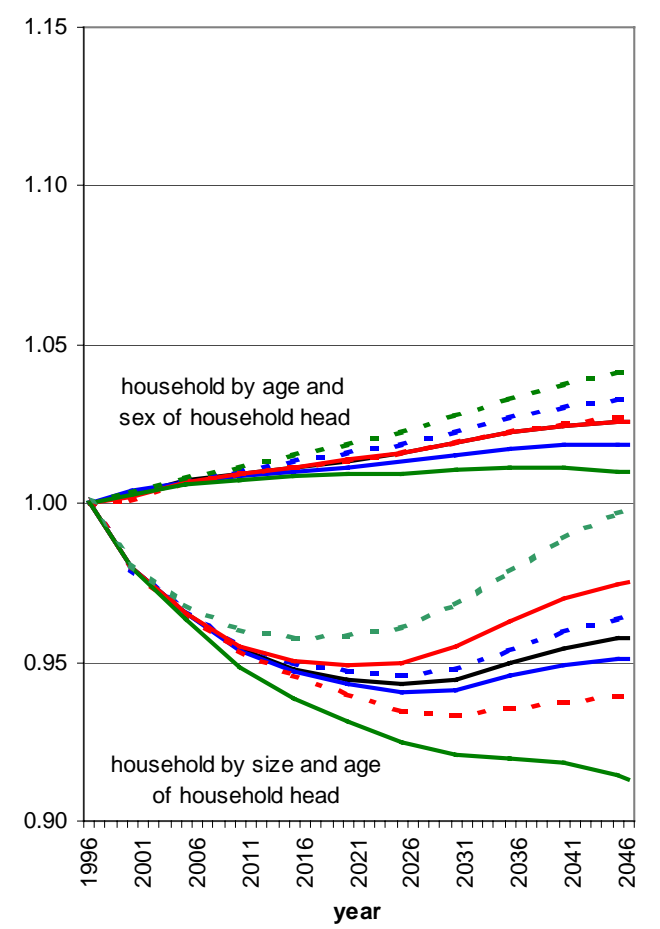

5c: Change in car use for alternative demographic scenarios by household size and age of household head and by household size and number of children relative to a projection by household size

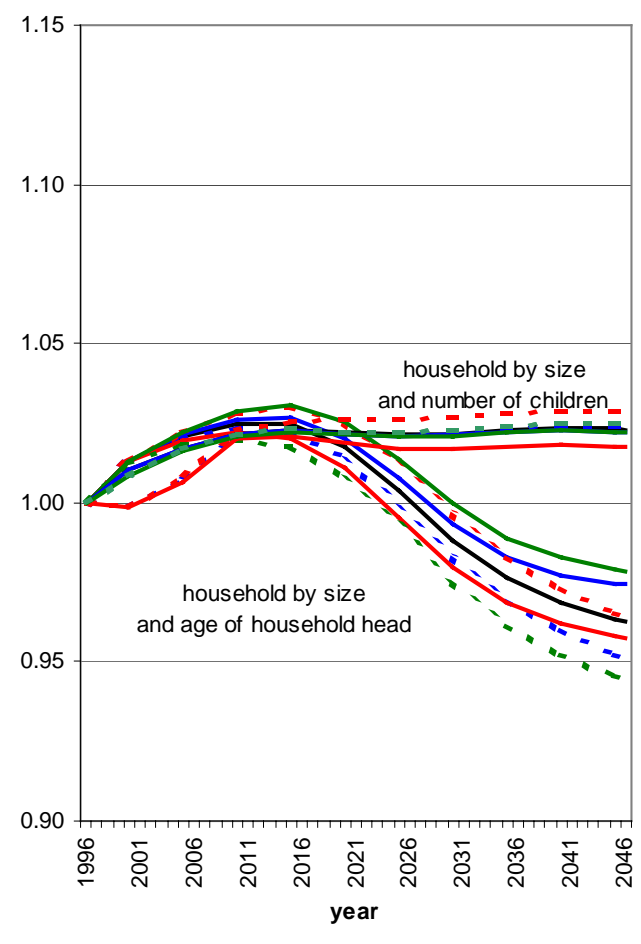


5d: Change in car use for alternative demographic scenarios by household size and age and sex of household head relative to a projection by household size and age of household head

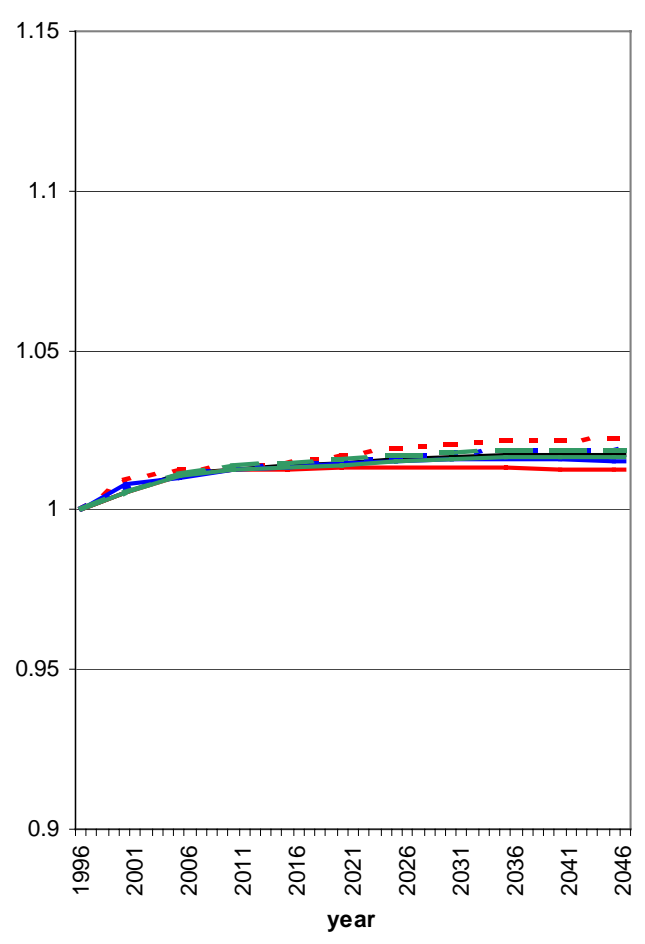

\begin{tabular}{ll} 
medium $_{\text {high fertility }}$ & high mortality $_{\text {high dissolution }}^{\text {n }}$ \\
- - - low mortality & - - - low fertility \\
- - - low dissolution & \\
\hline
\end{tabular}

5e: Change in car use for alternative demographic scenarios by household size and age and sex of household head relative to a projection by age and sex of household head

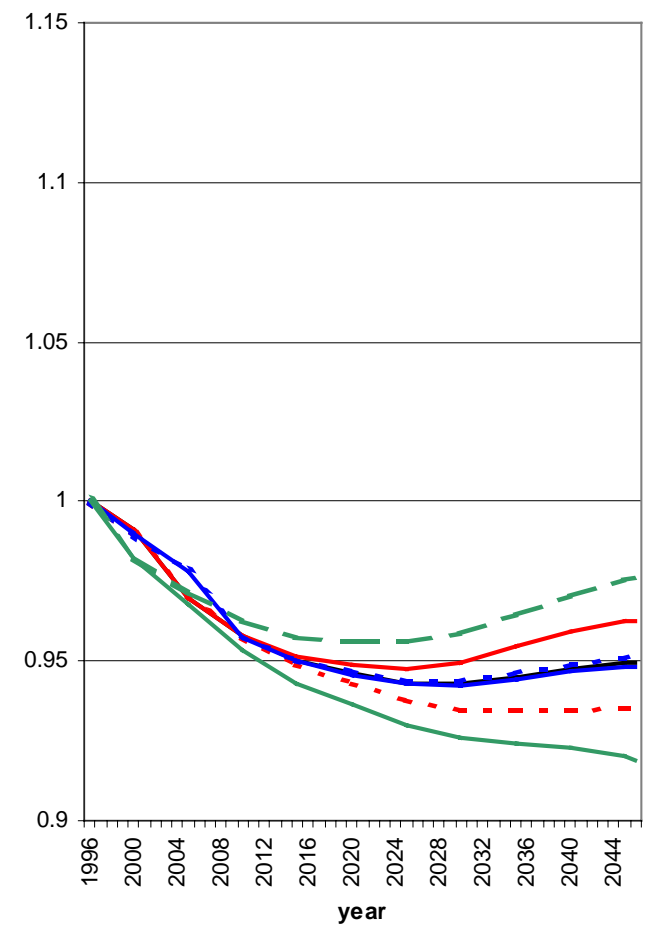




\section{Figure 6: Change in VKT per adult and energy efficiency}

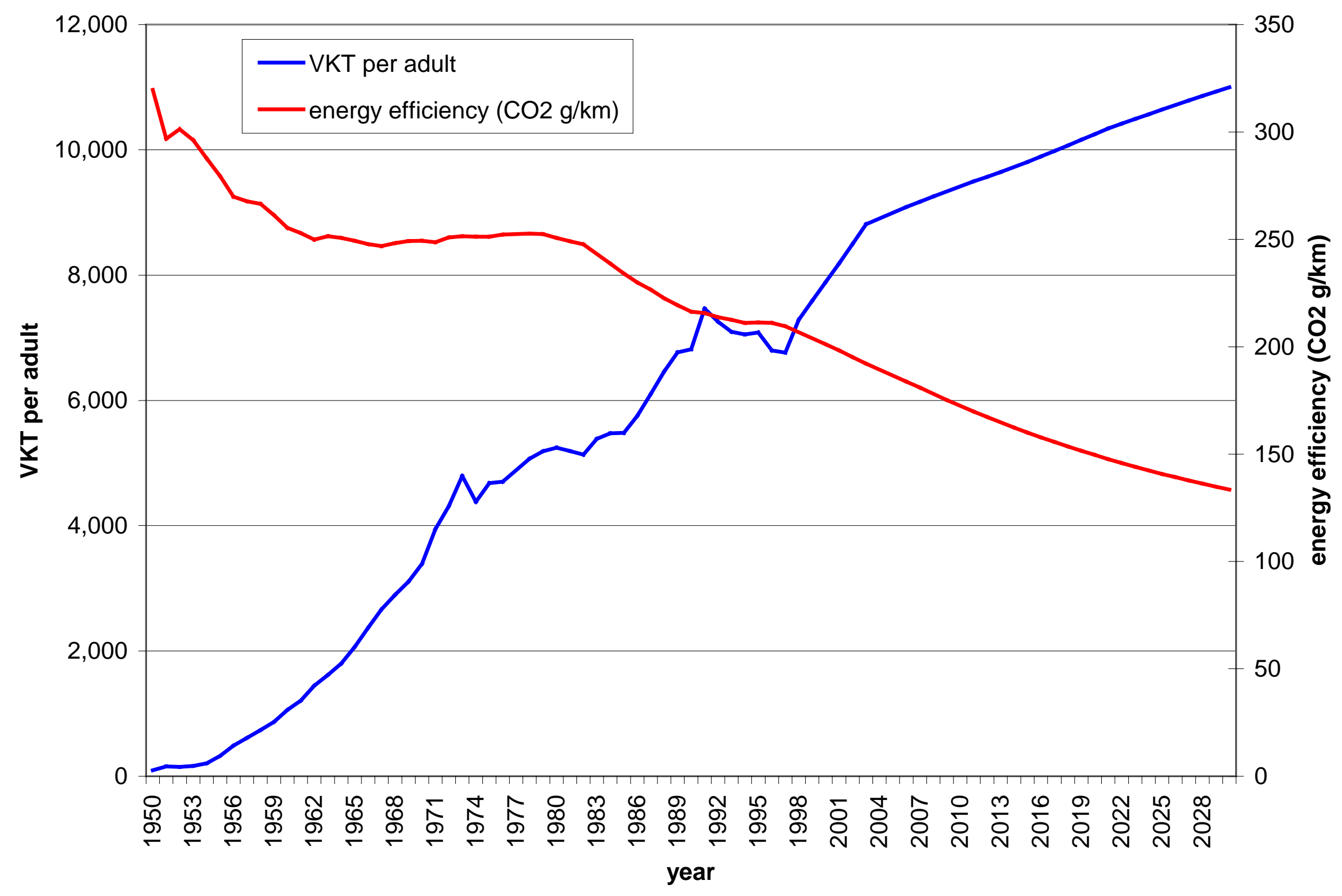

